\title{
Modeling the physical properties in the ISM of the low-metallicity galaxy NGC $4214^{\star}$
}

\author{
A. Dimaratos ${ }^{1}$, D. Cormier ${ }^{1}$, F. Bigiel ${ }^{1}$, and S. C. Madden ${ }^{2}$ \\ 1 Institut für theoretische Astrophysik, Zentrum für Astronomie der Universität Heidelberg, Albert-Ueberle Str. 2, 69120 Heidelberg, \\ Germany \\ e-mail: diane.cormier@zah.uni-heidelberg.de \\ ${ }^{2}$ Laboratoire AIM, CEA/DSM - CNRS - Université Paris Diderot, Irfu/Service d'Astrophysique, CEA Saclay, 91191 Gif-sur-Yvette, \\ France
}

Received 30 April 2015 / Accepted 12 June 2015

\section{ABSTRACT}

\begin{abstract}
We present a model for the interstellar medium of NGC 4214 with the objective to probe the physical conditions in the two main star-forming regions and their connection with the star formation activity of the galaxy. We used the spectral synthesis code CLOUDY to model an $\mathrm{H}$ II region and the associated photodissociation region (PDR) to reproduce the emission of mid- and far-infrared finestructure lines from the Spitzer and Herschel space telescopes for these two regions. Input parameters of the model, such as elemental abundances and star formation history, are guided by earlier studies of the galaxy, and we investigated the effect of the mode in which star formation takes place (bursty or continuous) on the line emission. Furthermore, we tested the effect of adding pressure support with magnetic fields and turbulence on the line predictions. We find that this model can satisfactorily predict (within a factor of $\sim 2$ ) all observed lines that originate from the ionized medium ([S IV] $10.5 \mu \mathrm{m},[\mathrm{Ne}$ III] $15.6 \mu \mathrm{m},[\mathrm{S} \mathrm{III]} 18.7 \mu \mathrm{m},[\mathrm{S} \mathrm{III]} 33.5 \mu \mathrm{m}$, and [O III] $88 \mu \mathrm{m}$ ), with the exception of [Ne II] $12.8 \mu \mathrm{m}$ and [N II] $122 \mu \mathrm{m}$, which may arise from a lower ionization medium. In the PDR, the [OI] $63 \mu \mathrm{m}$, [O I] $145 \mu \mathrm{m}$, and [C II] $157 \mu \mathrm{m}$ lines are matched within a factor of $\sim 5$ and work better when weak pressure support is added to the thermal pressure or when the PDR clouds are placed farther away from the H II regions and have covering factors lower than unity. Our models of the H II region agree with different evolutionary stages found in previous studies, with a more evolved, diffuse central region, and a younger, more compact southern region. However, the local PDR conditions are averaged out on the $175 \mathrm{pc}$ scales probed and do not reflect differences observed in the star formation properties of the two regions. Their increased porosity stands out as an intrinsic characteristic of the low-metallicity ISM, with the PDR covering factor tracing the evolution of the regions.
\end{abstract}

Key words. galaxies: dwarf - galaxies: ISM - galaxies: individual: NGC 4214 - HII regions - techniques: spectroscopic radiative transfer

\section{Introduction}

The physical state and structure of the interstellar medium (ISM) are important parameters for understanding the star formation in a galaxy. In a typical star-forming region, young massive stars are born and start to illuminate their parental cloud. UV photons ionize the surrounding medium, creating $\mathrm{H}$ II regions, while the transition to the neutral atomic or molecular phase occurs at higher visual extinction, where the material is more effectively shielded. Far-UV (FUV) photons control the chemical activity in these regions, namely the photodissociation region (PDR; Tielens \& Hollenbach 1985). By studying the latter, we can investigate the conditions of the molecular clouds, which in turn will be potential sites for the next episode of star formation.

How does the propagation of radiation and the ISM composition affect ISM observables in low-metallicity galaxies? Addressing this question is important to understand the evolution of low-metallicity galaxies, which undergo more bursty star formation than normal galaxies. Nearby star-forming dwarf galaxies present distinct observational signatures compared to well-studied disk galaxies. Dwarfs are usually metal poor, $\mathrm{HI}$ rich, and molecule poor as a result of large-scale photodissociation (e.g., Kunth \& Östlin 2000; Hunter et al. 2012; Schruba et al. 2012). Mid-IR (MIR) and far-IR (FIR) observations have

\footnotetext{
* Based on observations performed with Spitzer and Herschel.
}

revealed bright atomic lines from $\mathrm{H}$ II regions ([S III], [Ne III], [Ne II], [O III], etc.) and PDRs ([C II], [O I] ) (e.g., Hunter et al. 2001; Madden et al. 2006; Wu et al. 2008; Hunt et al. 2010; Cormier et al. 2015). Their spectral energy distributions (SEDs) are also different from spiral and elliptical galaxies and indicative of altered dust properties, with a relatively low abundance of polycyclic aromatic hydrocarbons (PAHs) and perhaps a different dust composition (e.g., Madden et al. 2006; Galliano et al. 2008; Rémy-Ruyer et al. 2013). It is still unknown, however, whether these differences between dwarf and disk galaxies are the direct result of recent star formation activity shaping the ISM or instead a consequence of the low-metallicity ISM that is independent of star formation activity. To answer this, one needs to observe tracers of the interplay between the ISM and various stages of star formation activity. While there are now a number of important studies available on PDR properties modeling FIR lines on large scales in various extragalactic environments (e.g., Kaufman et al. 2006; Vasta et al. 2010; Graciá-Carpio et al. 2011; Cormier et al. 2012; Parkin et al. 2013) or in our Galaxy under solar-metallicity conditions (e.g., Cubick et al. 2008; Bernard-Salas et al. 2012, 2015), only a few studies are published on individual extragalactic regions (Mookerjea et al. 2011; Lebouteiller et al. 2012). Of particular interest are dwarf galaxies, where the effect due to radiative feedback is expected to be most significant. The goal of this paper is to investigate how 


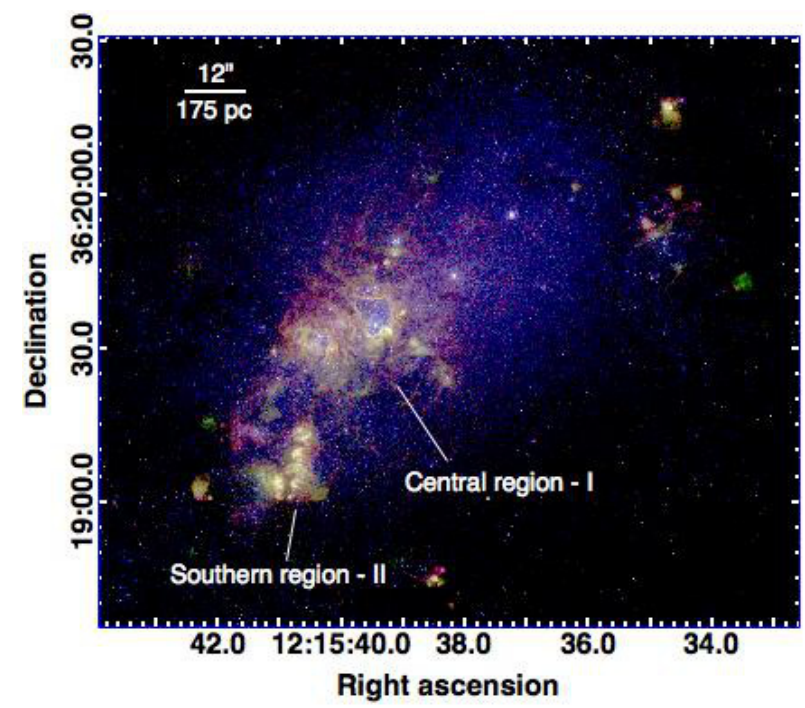

Fig. 1. Three-color image of NGC 4214 using the HST WFC3 filters $F 438 W$ (B, blue), F502N ([O III], green), and F657N (H $\alpha+[\mathrm{N} \mathrm{II}]$, red), downloaded from the Hubble Legacy Archive (http://hla.stsci . $\mathrm{edu} /)$.

the low-metallicity ISM reacts under the effects of star formation in regions that have undergone different histories. The nearby low-metallicity galaxy NGC 4214 provides an excellent environment to perform this experiment because it has well-separated star-forming centers, one hosting a super star cluster, which allows us to study the effects of extreme star-forming conditions on the surrounding ISM.

NGC 4214 is a nearby irregular galaxy located $3 \mathrm{Mpc}$ away (Dalcanton et al. 2009) with a metallicity of $\sim 0.3 Z_{\odot}$ (Kobulnicky \& Skillman 1996) and a wealth of ancillary data. It shows various morphological characteristics such as H I holes and shells and a spiral pattern (McIntyre 1998). NGC 4214 is known to host two main, well-defined star-forming regions with recent activity (Fig. 1). The largest of the two regions is found in the center of the galaxy (also referred to as NW or region I) and contains several clusters, including a super star cluster, while the second region is found to the southeast (also referred to as SE or region II) and is younger and more compact. Using near-IR, optical, and UV data, several studies have constrained the ages of the clusters in the two main regions, which show evidence for recent star formation (Úbeda et al. 2007a; Sollima et al. 2013, 2014). Schruba et al. (2012) have measured the ongoing SFR of NGC 4214 to be $0.12 M_{\odot} \mathrm{yr}^{-1}$. The galaxy seems to have maintained its star formation in the past $10 \mathrm{Gyr}$ at an average rate of $\sim 0.02 M_{\odot} \mathrm{yr}^{-1}$, with a prolonged star formation episode that occurred about $3 \mathrm{Gyr}$ ago and several shorter bursty events within the past Gyr at a rate of $0.05-0.12 M_{\odot} \mathrm{yr}^{-1}$ (McQuinn et al. 2010; Williams et al. 2011).

In this paper, we present observations of MIR and FIR finestructure cooling lines in NGC 4214, which provide key diagnostics of the physical conditions of the ISM. We focus our analysis on the two main star-forming complexes. The line emission is analyzed with radiative transfer models to characterize the ISM conditions. We take into account directly observed star formation histories and explore how they affect the IR line emission. Photometry is used for the energy budget of the models. The structure of this paper is the following: Sect. 2 describes the data, Sect. 3 describes the model, and the results are presented in Sect. 4. We summarize and discuss our results in Sect. 5.
Table 1. List of Herschel and Spitzer observations.

\begin{tabular}{|c|c|}
\hline & Herschel data \\
\hline Instrument & Observation Identification number (OBSID) \\
\hline PACS phot. & $\begin{array}{l}\text { 1342211803, 1342211804, 1342211805, } \\
1342211806\end{array}$ \\
\hline PACS spec. & $\begin{array}{l}\text { 1342187843, 1342187844, 1342187845, } \\
1342188034,1342188035,1342188036\end{array}$ \\
\hline Instrument & $\begin{array}{c}\text { Spitzer data } \\
\text { Astronomical Observation Request (AOR) }\end{array}$ \\
\hline $\begin{array}{l}\text { MIPS } 24 \mu \mathrm{m} \\
\text { IRS Short-High }\end{array}$ & $22652672,22652928,22710528,22710784$ \\
\hline On-source: & $10426368,10426624,10426880,10427136$ \\
\hline Background: & $\begin{array}{l}\text { 13728256, 13729792, 13730304, 13730816, } \\
\text { 13733120, 13762304, 13767424, 13767936, } \\
13768448,13768704,13769728,13770496, \\
13773568\end{array}$ \\
\hline IRS Long-High & \\
\hline On-source: & $\begin{array}{l}10424832,10425088,10425344,10425600, \\
10425856,10426112,10427392\end{array}$ \\
\hline Background: & $\begin{array}{l}\text { 13728768, 13729792, 13730304, 13730816, } \\
\text { 13733120, 13763328, 13764352, 13765376, } \\
\text { 13767424, 13767936, 13768448, 13768704, } \\
13769728,13770496,13773568\end{array}$ \\
\hline
\end{tabular}

\section{Data}

\subsection{Herschel data}

We used observations of NGC 4214 obtained by the PACS instrument (Poglitsch et al. 2010) onboard the Herschel Space Observatory (Pilbratt et al. 2010) as part of the Dwarf Galaxy Survey (Madden et al. 2013). The list of observations can be found in Table 1 . The photometry data at $70 \mu \mathrm{m}, 100 \mu \mathrm{m}$, and $160 \mu \mathrm{m}$, with respective beam sizes (FWHM) of 5.6", 6.7", and $11.3^{\prime \prime}$, were published by Rémy-Ruyer et al. (2013). These bands cover the peak of the SED originating from the reprocessed stellar light by the dust. The spectroscopy comprises observations of the [O III] $88 \mu \mathrm{m}$ and [N II] $122 \mu \mathrm{m}$ lines, which trace the ionized gas, as well as the [C II] $157 \mu \mathrm{m}$, [O I] $63 \mu \mathrm{m}$, and [OI] $145 \mu \mathrm{m}$ lines, which trace the PDR. The data consist of small mappings of $5 \times 5$ rasters separated by $\sim 16^{\prime \prime}$ for [O III] $88 \mu \mathrm{m}$ and [O I] $63 \mu \mathrm{m}$ and $3 \times 3$ rasters separated by $\sim 24^{\prime \prime}$ for the other lines, ensuring a uniform coverage of $1.6^{\prime} \times 1.6^{\prime}$. Originally presented in Cormier et al. (2010), the PACS spectral data were re-processed with the reduction and analysis software HIPE user release v.11 (Ott 2010) and PACSman v.3.5 (Lebouteiller et al. 2012). With the improved calibration and definition of the regions, flux maps are globally consistent with those published in Cormier et al. (2010) and line ratios agree within $30 \%$. Flux maps of the [C II] $157 \mu \mathrm{m}$ and [O I] $63 \mu \mathrm{m}$ lines are shown in Fig. 2. The associated error maps include data and line-fitting uncertainties, but not calibration uncertainties, which are on the order of $15 \%$. The FWHM is $9.5^{\prime \prime}$ below $100 \mu \mathrm{m}$ and $10^{\prime \prime}, 11^{\prime \prime}, 12^{\prime \prime}$ at $122 \mu \mathrm{m}, 145 \mu \mathrm{m}, 160 \mu \mathrm{m}$, respectively. All maps were convolved to the [C II] $157 \mu \mathrm{m}$ resolution of $\sim 12^{\prime \prime}$, which at the distance of NGC 4214 corresponds to a physical scale of $175 \mathrm{pc}$. In both the photometry and spectroscopy data sets, the convolutions were performed using kernels provided by Aniano et al. $(2011)^{1}$.

\subsection{Spitzer data}

NGC 4214 was observed with the three instruments onboard the Spitzer Space Telescope (Werner et al. 2004). We used the

1 http://www.astro.princeton. edu/ ganiano/Kernels/ 


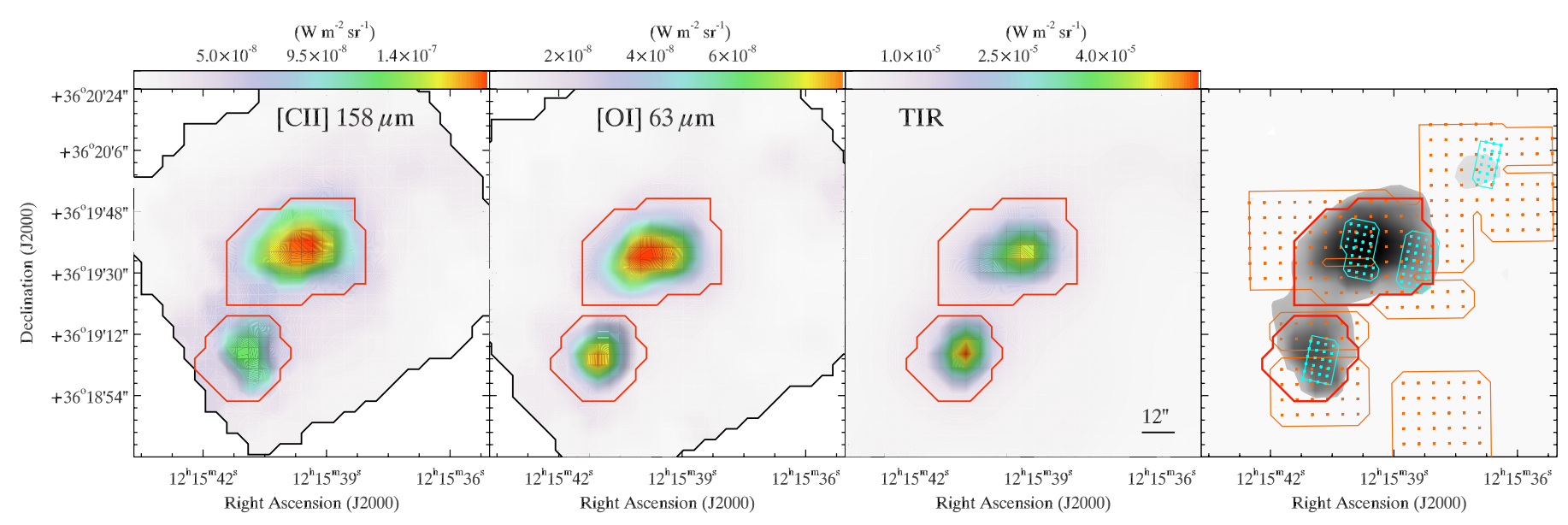

Fig. 2. Maps of the [C II] $157 \mu \mathrm{m}$, [O I] $63 \mu \mathrm{m}$, and TIR emission in NGC 4214. Units are $\mathrm{W} \mathrm{m}^{-2} \mathrm{sr}^{-1}$. The two star-forming regions, as defined in Sect. 2.4, are outlined with red contours. The right panel shows the Spitzer IRS mapping strategy. Orange: Long-High module coverage; cyan: Short-High module coverage; gray background: [C II] $157 \mu$ m map.

MIPS $24 \mu \mathrm{m}$ observations obtained within the Local Volume Legacy Survey (Dale et al. 2009) that were processed by Bendo et al. (2012). The MIPS $24 \mu \mathrm{m}$ map, which has an original FWHM of 5.9", was convolved to a resolution of $\sim 12^{\prime \prime}$ to match that of the PACS data.

The IRS observations (program ID 3177, PI. Skillman) consist of small mappings of the two main star-forming regions in high-resolution mode (Houck et al. 2004). We extracted the data from the Spitzer Heritage Archive (see Table 1) and processed them with the software CUBISM v1.8 (Smith et al. 2007). We used the default mapping procedure and bad pixel removal to produce spectral cubes with pixel sizes $2.26^{\prime \prime}$ for the ShortHigh module and $4.46^{\prime \prime}$ for the Long-High module. We then created surface brightness maps for all spectral lines of interest [S IV] $10.5 \mu \mathrm{m}$, [Ne II] $12.8 \mu \mathrm{m}$, [Ne III] $15.6 \mu \mathrm{m}$, [S III] $18.7 \mu \mathrm{m}$, [S III] $33.5 \mu \mathrm{m}$, which all trace $\mathrm{H}_{\text {II }}$ regions - in the following way: for each pixel of the cube, we extracted the signal within a range of $\pm 0.7 \mu \mathrm{m}$ around the line and fit a polynomial of order two for the baseline and a Gaussian for the line with the IDL routine mpfit. For a more stable fit, the peak of the Gaussian is required to be positive, the position of the peak is expected within one instrumental FWHM of the rest wavelength, and the width is limited to the instrumental resolution $(R=600)$. Finally, we added random noise and iterated the fit 300 times to estimate the best-fit parameters as the median of the resulting parameters and the error on those parameters as the standard deviation. Error maps again include data and line-fitting uncertainties, but not calibration uncertainties, which are on the order of $5 \%$. The coverage of the star-forming regions of the galaxy in the IRS maps is only partial, as shown in Fig. 2. No integrated values for the flux of the whole regions could be retrieved. To obtain a representative value for the line flux in each region, we regridded the IRS maps to that of the [O III] $88 \mu \mathrm{m}$ map. Then we selected the pixels that appear in both maps and scaled the emission of these pixels to the [O III] $88 \mu \mathrm{m}$ line to infer the corresponding line fluxes for the star-forming regions as a whole.

We focus on these selected IRS lines because they are among the brightest MIR fine-structure cooling lines and can be used as reliable diagnostics of the physical conditions in $\mathrm{H}$ II regions. In general, the intensity or luminosity ratio of two lines of the same element but different ionization level is indicative of the radiation field hardness. Such diagnostics are the [Ne III] $15.6 \mu \mathrm{m} /[\mathrm{Ne}$ II] $12.8 \mu \mathrm{m}$ or the [S IV] $10.5 \mu \mathrm{m} /$ [S III] $18.7 \mu \mathrm{m}$ ratios (e.g., Verma et al. 2003), which are insensitive to the density because of their high critical densities (see Table 2). Accordingly, species of the same ionization level but different transition are indicative of the electron density as a result of the different critical densities for each transition (Osterbrock \& Ferland 2006). Examples are the [S III] $18.7 \mu \mathrm{m} /[\mathrm{S} \mathrm{III}] 33.5 \mu \mathrm{m},[\mathrm{Ne}$ III] $15.6 \mu \mathrm{m} /[\mathrm{Ne}$ III] $36.0 \mu \mathrm{m}$, or [N II] $122 \mu \mathrm{m} /[\mathrm{N}$ II] $205 \mu \mathrm{m}$ ratios (e.g., Rubin et al. 1994). These diagnostics are insensitive to the temperature inside the $\mathrm{H}$ II region. Unfortunately, the [Ne III] $36.0 \mu \mathrm{m}$ and [N II] $205 \mu \mathrm{m}$ lines fall at the edge of the IRS and PACS wavelength ranges, respectively, where the spectra are too noisy to detect or derive a reliable line ratio for the two star-forming regions. Therefore we relied on the $[\mathrm{S} \mathrm{III]}$ lines to probe the electron density.

\subsection{Total infrared luminosity map}

To construct a total infrared (TIR) luminosity map of the galaxy, we combined the MIPS $24 \mu \mathrm{m}$ and PACS 70, 100, and $160 \mu \mathrm{m}$ data, following Galametz et al. (2013):

$L_{\mathrm{TIR}}=\int_{3 \mu \mathrm{m}}^{1100 \mu \mathrm{m}} L_{v} \mathrm{~d} v=\sum c_{i} L_{i}$.

We used the values of the coefficients, $c_{i}$, from Table 3 of their paper: $\left[c_{24}, c_{70}, c_{100}, c_{160}\right]=[2.064,0.539,0.277,0.938]$. This method, although slightly less accurate than a direct integration of a well-sampled SED, does not require degrading the resolution of our data beyond the PACS $160 \mu \mathrm{m}$ beam and is sufficient for our modeling purposes to estimate the energy budget in the star-forming regions. The $L_{\mathrm{TIR}}$ map is shown in Fig. 2.

\subsection{Defining the star-forming regions}

To define the apertures for the main star-forming regions, we set a threshold for the signal-to-noise ratio $(\mathrm{S} / \mathrm{N})$ equal to 5 in each individual PACS $70 \mu \mathrm{m}, 100 \mu \mathrm{m}$, and $160 \mu \mathrm{m}$ photometry and PACS spectral map. We masked all pixels below this $\mathrm{S} / \mathrm{N}$ and drew the contours, which include all the remaining unmasked pixels, separately for the photometry and the spectroscopy maps. Because the emission in the photometry maps is more extended, we kept the contours from the photometry and used these apertures throughout the analysis to define the two star-forming regions, as shown in Fig. 2. This means that pixels in the spectroscopy maps that are below the $\mathrm{S} / \mathrm{N}$ threshold but within the 
Table 2. Observed MIR and FIR fluxes for the line and broadband emission.

\begin{tabular}{|c|c|c|c|c|c|}
\hline \multirow[t]{2}{*}{ Line } & \multirow{2}{*}{$\begin{array}{l}\text { Wavelength } \\
\qquad(\mu \mathrm{m})\end{array}$} & \multicolumn{2}{|c|}{$\begin{array}{l}\text { Flux } \pm \text { uncertainty } \\
\left(\times 10^{-16} \mathrm{~W} \mathrm{~m}^{-2}\right)\end{array}$} & \multirow{2}{*}{$\begin{array}{l}\text { Critical density } \\
\quad\left(\mathrm{cm}^{-3}\right)\end{array}$} & \multirow{2}{*}{$\begin{array}{c}\text { Ionization } \\
\text { potential } \\
(\mathrm{eV})\end{array}$} \\
\hline & & Region I & Region II & & \\
\hline [S IV] & 10.51 & $5.68 \pm 0.21$ & $8.40 \pm 0.10$ & $5 \times 10^{4}[\mathrm{e}]$ & 34.79 \\
\hline$[\mathrm{Ne} \mathrm{II}]$ & 12.81 & $8.98 \pm 0.22$ & $4.13 \pm 0.11$ & $7 \times 10^{5}[\mathrm{e}]$ & 21.56 \\
\hline$[\mathrm{Ne}$ III] & 15.56 & $18.70 \pm 0.14$ & $14.25 \pm 0.08$ & $3 \times 10^{5}[\mathrm{e}]$ & 40.96 \\
\hline$[\mathrm{S}$ III $]$ & 18.71 & $11.78 \pm 0.20$ & $6.85 \pm 0.08$ & $2 \times 10^{4}[\mathrm{e}]$ & 23.34 \\
\hline [S III] & 33.48 & $18.71 \pm 0.27$ & $8.20 \pm 0.12$ & $7 \times 10^{3}[\mathrm{e}]$ & 23.34 \\
\hline$[\mathrm{O} \mathrm{I}]$ & 63.18 & $10.11 \pm 0.35$ & $4.06 \pm 0.21$ & $5 \times 10^{5}[\mathrm{H}]$ & - \\
\hline [O III] & 88.36 & $31.86 \pm 0.62$ & $13.50 \pm 0.40$ & $5 \times 10^{2}[\mathrm{e}]$ & 35.12 \\
\hline$[\mathrm{N}$ II $]$ & 121.90 & $0.44 \pm 0.20$ & $0.14 \pm 0.08$ & $3 \times 10^{2}[\mathrm{e}]$ & 14.53 \\
\hline$[\mathrm{O} \mathrm{I}]$ & 145.52 & $0.65 \pm 0.09$ & $0.32 \pm 0.07$ & $1 \times 10^{5}[\mathrm{H}]$ & - \\
\hline$[\mathrm{C}$ II $]$ & 157.74 & $26.34 \pm 0.33$ & $10.05 \pm 0.21$ & $50[\mathrm{e}], 3 \times 10^{3}[\mathrm{H}]$ & 11.26 \\
\hline & \multirow[t]{2}{*}{ Broadband } & \multirow{2}{*}{$\begin{array}{l}\text { Wavelength } \\
\qquad(\mu \mathrm{m})\end{array}$} & \multicolumn{2}{|c|}{$\begin{array}{c}\text { Flux density } \pm \text { uncertainty } \\
(\mathrm{Jy})\end{array}$} & \\
\hline & & & Region & Region II & \\
\hline & \multicolumn{2}{|l|}{ MIPS } & $0.67 \pm 0.01$ & $0.48 \pm 0.01$ & \\
\hline & PACS & 70 & $7.36 \pm 0$ & $3.72 \pm 0.12$ & \\
\hline & PACS & 10 & $7.91 \pm($ & $4.10 \pm 0.11$ & \\
\hline & PACS & 16 & $6.07 \pm($ & $3.23 \pm 0.06$ & \\
\hline & $L_{\mathrm{TIR}}(\mathrm{e}$ & $3-11$ & $5.25 \times$ & $3.02 \times 10^{41}$ & \\
\hline
\end{tabular}

Notes. Uncertainties on the fluxes include data and line-fitting uncertainties, but not calibration uncertainties, which are on the order of $5 \%$ for the Spitzer lines and 15\% for the Herschel lines. Critical density and ionization potential values are taken from Cormier et al. (2012). Critical densities are noted $[\mathrm{e}]$ for collisions with electrons and $[\mathrm{H}]$ for collisions with hydrogen atoms.

Table 3. Elemental abundances in NGC 4214.

\begin{tabular}{lccc}
\hline \hline Abundance & Region I & Region II & Solar value \\
\hline$[\mathrm{O} / \mathrm{H}]$ & $-3.795 \pm 0.05$ & $-3.64 \pm 0.04$ & $-3.31 \pm 0.05$ \\
{$[\mathrm{~S} / \mathrm{H}]$} & $-5.380 \pm 0.06$ & $-5.21 \pm 0.06$ & $-4.88 \pm 0.03$ \\
{$[\mathrm{~N} / \mathrm{H}]$} & $-5.094 \pm 1.00$ & $-5.02 \pm 0.10$ & $-4.17 \pm 0.05$ \\
{$[\mathrm{Ne} / \mathrm{H}]$} & $-4.535 \pm 0.11$ & $-4.51 \pm 0.08$ & $-4.07 \pm 0.10$ \\
{$[\mathrm{C} / \mathrm{H}]$} & $-4.295 \pm 0.30$ & $-4.14 \pm 0.30$ & $-3.57 \pm 0.05$ \\
\hline
\end{tabular}

Notes. Values (in logarithmic scale) for NGC 4214 are taken from Kobulnicky \& Skillman (1996) and solar values from Asplund et al. (2009).

region contours are still counted. The fluxes and uncertainties for the line and TIR emission were measured taking into account all pixels in each region. They are reported in Table 2. The ISM emission (gas and dust) peaks in these two regions, and most of the line fluxes are twice as high in the central region as in the southern region, except for [Ne III] $15.6 \mu \mathrm{m}$ (factor 1.3) and [S IV] $10.5 \mu \mathrm{m}$ (factor 0.8). This hints at different physical conditions in the two regions, which we investigate with radiative transfer models.

\section{Description of the model}

\subsection{Model geometry and strategy}

Our objective is to characterize the physical conditions of the ISM phases from which the IR emission arises in NGC 4214. To that end, we used the spectral synthesis code CLOUDY v.13, last described by Ferland et al. (2013). We performed a multiphase detailed modeling of the ISM for which we combined line and continuum emission, following the method described in Cormier et al. (2012). We considered the two main star-forming regions of NGC 4214: the most evolved central region (NW-I) and the southern region (SE-II). Here we present the main aspects of the model and how it is applied to each region. The components/ ISM phases of the model are

1. a central source of radiation,

2. an ionized medium component (H II region) that surrounds the central source,

3. a neutral medium (PDR) surrounding the H II region.

This method assumes a single radiation source responsible for the observed SED of the studied region. In other words, we took all of the different sources (star clusters) and the surrounding clouds from which they have formed and represented them with one central source and one surrounding cloud. We thus targeted the integrated properties of each region. In practice, we mixed components of the ISM that have different composition and properties and blend them in a single system. The applied geometry is spherical. The source is in the center and the illuminated face of the cloud lies at a certain distance that we call inner radius. In our case, the effective geometry is one-dimensional plane-parallel because the cloud forms a thin shell and its distance from the radiation source is large.

The radiation source, representative of the stars that populate the clusters of the star-forming region, illuminates a cloud of dust and gas. It controls the ionization parameter, $U$, which characterizes the field and is defined as the ratio of the incident ionizing photon density to the hydrogen density. Hard UV photons from the source ionize hydrogen and form the H II region. As this radiation is transmitted through the cloud, it is attenuated and thus becomes softer, which decreases its influence on ionization. However, it still controls the processes further in the cloud (in the PDR).

The adopted strategy is to treat the H II region first and then use the HII region parameters as input for the PDR modeling. This allows for a self-consistent approach (Abel et al. 2005), which is usually not directly available in standard PDR codes (see Röllig et al. 2007 for a comparison of PDR codes), and is important to accurately derive the radiation field that impinges 
on the PDR. We first ran the simulation until the end of the $\mathrm{H}$ II region, choosing to stop the simulation when the (electron) temperature reached $500 \mathrm{~K}$ to ensure that the model had transitioned to the atomic phase. We iterated to optimize our parameters so that they matched the observed emission of known H II region-diagnostic lines ([S IV] $10.5 \mu \mathrm{m}$, [Ne II] $12.8 \mu \mathrm{m}$, [Ne III] $15.6 \mu \mathrm{m}$, [S III] $18.7 \mu \mathrm{m}$, [S III] $33.5 \mu \mathrm{m}$, [O III] $88 \mu \mathrm{m}$, and [N II] $122 \mu \mathrm{m}$ ). Then we fed the result of this model to the PDR and compared the predictions to the remaining three PDR lines observed: [OI] $63 \mu \mathrm{m}$, [O I] $145 \mu \mathrm{m}$, and [C II] $157 \mu \mathrm{m}$, choosing a visual extinction of 10 mag as the stopping criterion. At this point, the gas temperature had fallen to roughly $10 \mathrm{~K}$.

\subsection{Model parameters}

We constrained the properties of the star-forming regions by varying some of the parameters that control the physics of the models while keeping others fixed. The main parameters that we consider are

1. a radiation field source: shape, age, luminosity (varied),

2. the hydrogen density of the ISM, $n_{\mathrm{H}}$ (varied),

3. the ISM gas elemental abundances (fixed),

4. the inner radius, $r_{\text {in }}$ (varied),

5. the magnetic field, $B$ (fixed),

6. the turbulent velocity, $v_{\text {turb }}$ (fixed).

Parameters that are fixed were set to values from the literature. The other parameters were varied inside a range whose width reflects the dispersion of published measurements or of the data. The main parameter of interest for this study is the radiation field, which was varied within a range guided by studies of the star formation history.

\subsubsection{Hydrogen density $\left(n_{H}\right)$}

We performed our simulations assuming pressure equilibrium. As the model proceeds through consecutive zones of the cloud, it keeps the pressure constant. Thus, the density of the medium varies to satisfy this equilibrium. The initial density that we specified in the models is the density at the illuminated face of the cloud, where the H II region starts. The initial values and the range we probed are motivated by the observed [S III] $18.7 \mu \mathrm{m} /[\mathrm{S} \mathrm{III]} 33.5 \mu \mathrm{m}$ ratio in the $\mathrm{H}$ II region, which is known to be sensitive to the electron density in the range $10^{2}-10^{4} \mathrm{~cm}^{-3}$ (Rubin et al. 1994). We therefore let the initial density vary in the range $100-300 \mathrm{~cm}^{-3}$ for the central region and $300-600 \mathrm{~cm}^{-3}$ for the southern region with a common step of $25 \mathrm{~cm}^{-3}$. By iterating this procedure, we constrained the best values for the density at the beginning of the $\mathrm{H}$ II region.

\subsubsection{Inner radius $\left(r_{\text {in }}\right)$}

In our spherical geometry, the source is at the center and is surrounded by a cloud. The illuminated face of the cloud lies at a certain distance $r_{\text {in }}$. This is not a strictly physically constrained parameter because the setup we used does not realistically model each cluster, but instead tries to mimic a whole region and reproduce its emission. The variation of this radius changes the photons flux and thus is expected to affect our results. We let $r_{\text {in }}$ vary from 1 to $100 \mathrm{pc}$ for both regions.

\subsubsection{Elemental abundances}

Elemental abundances in the models were set to the observed values for oxygen, sulfur, nitrogen, and neon, taken from Kobulnicky \& Skillman (1996). Some measurements partially cover our defined regions, and we adopted them as representative. Exclusively for carbon, we scaled its abundance according to the study on the dependence of $\log (C / O)$ on metallicity by Izotov \& Thuan (1999). For other elements, we used the default ISM composition of CLOUDY and scaled the abundances to our metallicity (1/3). The values used are indicated in Table 3.

\subsubsection{Radiation field - shape and energy}

We used the code STARBURST99 (Leitherer et al. 2010) to produce a stellar population spectrum that serves as input for our models. We chose a Kroupa initial mass function between 0.08 and $120 M_{\odot}$ (Kroupa 2001), as done in Andrews et al. (2013), and Padova asymptotic giant branch tracks with $Z=0.004$. As discussed above, we did not model each cluster individually, but we used integrated emission from the entire star-forming regions instead. We tried to be as close to the shape and intensity of the radiation field of the $\mathrm{H}$ II regions as possible. Motivated by the star formation history of the galaxy presented in McQuinn et al. (2010) and Williams et al. (2011), we tested the following cases:

- For the central region, we considered two limiting scenarios: (i) a single-burst star formation event and (ii) a continuous star formation model, with $S F R=0.07 M_{\odot} \mathrm{yr}^{-1}$. The ages of the clusters were varied within a range of (i) 1-20 Myr (in steps of $0.5 \mathrm{Myr}$ ) and (ii) $200-1000 \mathrm{Myr}$ (in steps of $200 \mathrm{Myr}$ ).

- For the southern region, we considered a single-burst event with an age that varied from 1 to $20 \mathrm{Myr}$ (in steps of $0.5 \mathrm{Myr}$ ).

A fixed mass of $10^{5} M_{\odot}$ (typical cluster mass in Sollima et al. 2013) was considered for the single bursts, where the stars are created at once (delta burst). Our value for the SFR $\left(0.07 M_{\odot} \mathrm{yr}^{-1}\right)$ is representative of the "average" rate at which this galaxy formed stars within the past 1 Gyr of its history. The ages of the clusters in both regions were guided by values from Úbeda et al. (2007a), Sollima et al. (2013, 2014). Úbeda et al. (2007a) found 2-7 Myr for region I, along with extended clusters in the same region with ages of 150 to $190 \mathrm{Myr}$. In region II, they found ages spreading around 2 Myr. Sollima et al. (2013, 2014) reported a larger spread in ages. In region I, they obtained ages around a median of $14 \mathrm{Myr}$, and for the more extended clusters the ages lie between 10 and 300 Myr. For region II, they found a median age of $\sim 20 \mathrm{Myr}$.

For the luminosity emitted from each region, we chose to use the TIR luminosity as a first approximation of the luminosity of the starburst. This choice implies the assumption that all radiation from the clusters in the region is reprocessed by the dust and thus emitted at longer wavelengths. In doing so, we kept in mind that there can be processes that we did not model (UV escape fraction or a diffuse ionized medium, for example) and that can contribute to this radiated energy (see Sect. 5).

\subsubsection{Magnetic field strength $(B)$ and turbulent velocity $\left(v_{\text {turb }}\right)$}

Magnetic fields and turbulence play an important role in structuring the ISM (e.g., McKee \& Ostriker 2007). When CLOUDY solves the pressure equilibrium for each zone of the modeled 
Table 4. Input parameters for the best-fitting models.

\begin{tabular}{lccc}
\hline \hline Parameter & \multicolumn{2}{c}{ Central region } & Southern region \\
& single burst & continuous & single burst \\
\hline Density $n_{\mathrm{H}}\left[\mathrm{cm}^{-3}\right]$ & 155 & 180 & 440 \\
Inner radius $r_{\text {in }}[\mathrm{pc}]$ & 85.4 & 62.1 & 22.3 \\
Stellar age $t[\mathrm{Myr}]$ & 4.1 & 440 & 3.9 \\
Total luminosity $L\left[\mathrm{erg} \mathrm{s}^{-1}\right]$ & $1.15 \times 10^{42}$ & $1.91 \times 10^{42}$ & $5.25 \times 10^{41}$ \\
Magnetic field $B[\mu \mathrm{G}]$ & - & $-(30)$ & - \\
Turbulent velocity $v_{\text {turb }}\left[\mathrm{km} \mathrm{s}^{-1}\right]$ & 1.5 & $1.5(3,50)$ & 1.5 \\
\hline
\end{tabular}

Notes. Values are given at the illuminated face of the cloud. Values in parenthesis for the magnetic field strength and turbulent velocities are tested in Sect. 4.2.

cloud, a magnetic pressure term equal to $P_{\mathrm{B}}=\frac{B^{2}}{8 \pi}$ is included in the equation of state along with a turbulent pressure term equal to $P_{\text {turb }}=2.8 \times 10^{6} \cdot 3 \cdot\left(\frac{n_{\mathrm{H}}}{10^{5} \mathrm{~cm}^{-3}}\right)\left(\frac{v_{\text {turb }}}{1 \mathrm{~km} \mathrm{~s}^{-1}}\right)^{2}\left[\mathrm{~cm}^{-3} \mathrm{~K}\right]$, for isotropic turbulent motions, where $n_{\mathrm{H}}$ is the total hydrogen density and $v_{\text {turb }}$ is the turbulent velocity (see the HAZY documentation of CLOUDY for more information).

The magnetic field of NGC 4214 was measured by Kepley et al. (2011) using multiwavelength radio emission. The reported field strength in the center of the galaxy is $30 \mu \mathrm{G}$, and the pressure term due to this field has the same order of magnitude as the hot gas and the gravitational contributions. Since it is not well known how the observed magnetic field might affect our observed line intensities, we excluded it from our default models and tested one case with a magnetic field strength of $30 \mu \mathrm{G}$.

Another potential energy source to consider can arise from the dissipation of turbulence. Turbulent energy is converted into thermal energy as it cascades from large scales to small scales through dissipation. However, we did not resolve size scales for which we can measure this. CLOUDY does not attempt to model the dissipation mechanism, but assumes a simple thermal energy source based on line width. The turbulent velocity was set to a value of $1.5 \mathrm{~km} \mathrm{~s}^{-1}$ by default in our models, and we tested two other cases: one case with an intermediate turbulent velocity $\left(v_{\text {turb }}=3 \mathrm{~km} \mathrm{~s}^{-1}\right.$ or $\left.F W H M=5 \mathrm{~km} \mathrm{~s}^{-1}\right)$ that corresponds to the approximate line width observed in the $\mathrm{CO}(1-0)$ data by Walter et al. (2001), and one case with a high turbulent velocity $\left(v_{\text {turb }}=50 \mathrm{~km} \mathrm{~s}^{-1}\right)$ as found in the diffuse ionized gas by Wilcots \& Thurow (2001) and used also in Kepley et al. (2011).

Nevertheless, we explore the effects of excluding or including magnetic fields and turbulence in Sect. 4.2.

\subsection{Determination of best-fitting models}

We aim to converge on a unique parameter set that best describes the conditions of the regions. We first ran models for which we varied the parameters in a coarse grid to narrow down the parameter space, using ranges of values found in the literature to start with. We then used the optimization option of CLOUDY, which automatically varies the specified parameters in a finer grid to find the optimal solution.

We computed the average $\chi^{2}$, denoted $\bar{\chi}^{2}$, for each model by comparing the observed fluxes of [S IV] $10.5 \mu \mathrm{m}$, [Ne III] $15.6 \mu \mathrm{m}, \quad[\mathrm{S} \mathrm{III}] 18.7 \mu \mathrm{m}, \quad[\mathrm{S} \mathrm{III}] 33.5 \mu \mathrm{m}$, and [O III] $88 \mu \mathrm{m}$ to the fluxes predicted from the radiative transfer calculation. These lines are the most luminous and most strongly correlated with the $\mathrm{H}$ II region (as opposed to [N II] and [Ne II] which, from experience, can arise from other phases). We refer to these as the optimized lines in Table 5. The goodness of
Table 5. $\chi^{2}$ values for the two star-forming regions.

\begin{tabular}{|c|c|c|c|}
\hline \multirow{2}{*}{ H II region } & \multicolumn{2}{|c|}{ Central region } & \multirow{2}{*}{$\begin{array}{c}\text { Southern region } \\
\text { burst }\end{array}$} \\
\hline & burst & continuous & \\
\hline \multicolumn{4}{|c|}{ Individual $\chi^{2}$ values: } \\
\hline$[\mathrm{N}$ II $] 122 \mu \mathrm{m}$ & 12.58 & 44.63 & 82.56 \\
\hline [S III] $18.7 \mu \mathrm{m}$ & 2.24 & 2.38 & 37.69 \\
\hline$[\mathrm{S} \mathrm{III}] 33.5 \mu \mathrm{m}$ & 8.10 & 6.99 & 31.89 \\
\hline$[\mathrm{S}$ IV $] 10.5 \mu \mathrm{m}$ & 0.76 & 0.70 & 4.94 \\
\hline$[\mathrm{Ne}$ II $] 12.8 \mu \mathrm{m}$ & 14442.2 & 13842.6 & 58101.7 \\
\hline [Ne III] $15.6 \mu \mathrm{m}$ & 8.14 & 24.65 & 224.51 \\
\hline $\bar{\chi}^{2}$ (all ionic lines) & 2067.72 & 1988.85 & 8354.76 \\
\hline $\bar{\chi}^{2}$ (optimized lines) & 3.85 & 6.95 & 59.81 \\
\hline \multirow[t]{2}{*}{ PDR } & \multicolumn{2}{|c|}{ Central region } & Southern region \\
\hline & burst & continuous & burst \\
\hline \multicolumn{4}{|l|}{ Individual $\chi^{2}$ values: } \\
\hline$[\mathrm{C}$ II $] 157 \mu \mathrm{m}$ & 1.57 & 31.83 & 258.63 \\
\hline [O I] $63 \mu \mathrm{m}$ & 149.78 & 57.05 & 85.37 \\
\hline$[\mathrm{O} \mathrm{I}] 145 \mu \mathrm{m}$ & 34.14 & 11.83 & 21.27 \\
\hline $\bar{\chi}^{2}$ (all PDR lines) & 61.83 & 33.57 & 121.76 \\
\hline
\end{tabular}

the line emission fit is given by low $\bar{\chi}^{2}$ values. The optimization method of CLOUDY searches the minimum of $\bar{\chi}^{2}$ that is defined as

$\bar{\chi}^{2}=\frac{1}{n} \sum \chi_{i}^{2}=\frac{1}{n} \sum \frac{\left(M_{i}-O_{i}\right)^{2}}{\left(\min \left\{O_{i} ; M_{i}\right\} \times \sigma_{i}\right)^{2}}$,

where $n$ is the number of lines optimized and $\chi_{i}^{2}$ are the $\chi^{2}$ values of the individual optimized lines. $M_{i}$ and $O_{i}$ are the modeled and observed fluxes, and $\sigma_{i}$ is the fractional error on the observed flux (uncertainty/flux) with calibration uncertainties added in quadrature to the measured uncertainties described in Sect. 2. We have five observables (the ionic lines listed above) and varied four parameters (cluster age, source luminosity, hydrogen density, and inner radius).

\section{Results}

In this section we present the model results for the two regions according to the star formation histories considered. Parameters of the best-fitting models and their corresponding $\chi^{2}$ values are reported in Tables 4 and 5, respectively.

\subsection{Line emission}

\subsubsection{Central region (I): the single-burst model}

For the single-burst star formation event, the best-fitting model of the central region has the following parameters: burst 
Central region
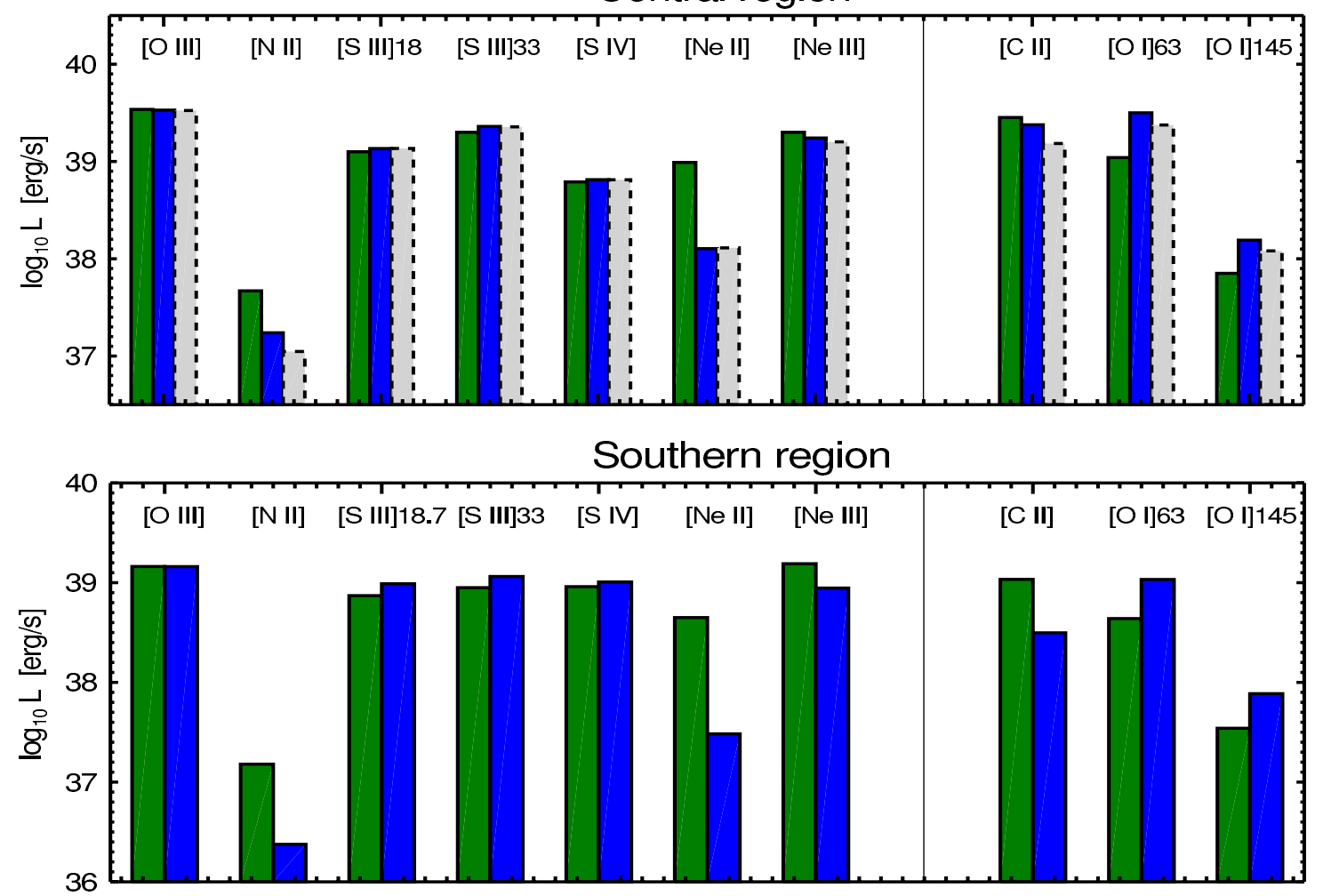

Fig. 3. Results for the central region (top panel) and for the southern region (bottom panel): line emission for the H II region (left side) and the PDR (right side), assuming pressure equilibrium. Green bars represent the observations, blue bars our single-burst model predictions, and gray bars with a dashed outline the continuous star formation model predictions.

age of $4.1 \mathrm{Myr}$ with luminosity $1.1 \times 10^{42} \mathrm{erg} \mathrm{s}^{-1}$, density of $155 \mathrm{~cm}^{-3}$, and $r_{\text {in }} \simeq 85 \mathrm{pc}$. The corresponding ionization parameter at the illuminating face of the cloud is $\log (U)=-2.7$. For the $\mathrm{H}$ II region, all optimized lines are matched within $\pm 30 \%$, and [Ne II] $12.8 \mu \mathrm{m}$ and [N II] $122 \mu \mathrm{m}$ are underpredicted by a factor of $\sim 8$ and 3 , respectively (see Fig. 3, blue bars in the top panel). In the PDR, the [O I] $145 \mu \mathrm{m}$ and $63 \mu \mathrm{m}$ lines are overpredicted by a factor of $\sim 2.5$, and the [C II] $157 \mu \mathrm{m}$ line is matched within $20 \%$. We note that [C II] emission can arise from both the neutral and the ionized phases of the ISM, with a potentially non-negligible contribution from the warm ionized medium as found in the Milky Way (Heiles 1994). The H II region of our best-fitting model contributes negligibly to the predicted [C II] $157 \mu \mathrm{m}$ and [O I] emission $(<3 \%)$. The ultraviolet radiation field strength $G_{0}$ inside the PDR is 455 in units of the equivalent Habing (1968) flux $\left(1 G_{0}=1.6 \times 10^{-3} \mathrm{erg} \mathrm{cm}^{-2} \mathrm{~s}^{-1}\right)$. The input luminosity required by the model, which all comes out as $L_{\mathrm{TIR}}$ in the PDR, is about twice as high as the observed $L_{\mathrm{TIR}}$ in that region.

These results represent the simplifying case that the recent starburst dominates the star formation in this central region, so the line emission can be explained with a single burst. The age of the burst in the model nicely agrees with the range of ages from Úbeda et al. (2007a) and is at the younger end of ages from Sollima et al. (2013, 2014).

\subsubsection{Central region (I): the continuous star formation model}

In the continuous star formation scenario, the best-fitting model of the central region has the following parameters: stellar age of $440 \mathrm{Myr}$ with luminosity $1.9 \times 10^{42} \mathrm{erg} \mathrm{s}^{-1}$, density of $180 \mathrm{~cm}^{-3}$, and $r_{\text {in }} \simeq 62$ pc. The ionization parameter is $\log (U)=-2.5$. Line predictions for the HII region and the
PDR from this model solution are shown in Fig. 3 (gray bars). For all optimized lines ([O III] $88 \mu \mathrm{m}$, [S III] 18.7 and $33.5 \mu \mathrm{m}$, [S IV] $10.5 \mu \mathrm{m}$, and [Ne III] $15.6 \mu \mathrm{m}$ ), the model matches the observations within $\pm 20 \%$. The two other ionic lines, [Ne II] $12.8 \mu \mathrm{m}$ and [N II] $122 \mu \mathrm{m}$, are underpredicted by a factor of $\sim 7$ and 4 . In the PDR, [C II] and the [OI] lines are matched within a factor of $\sim 2$. The contribution of the $\mathrm{H}$ II region to the predicted PDR emission is only $1 \%$. We find $G_{0} \simeq 1.2 \times 10^{3}$, which is higher than in the single-burst case. The luminosity of the model exceeds the observed $L_{\mathrm{TIR}}$ by a factor of 3.5.

The results represent the simplifying case of continuous star formation dominating this region, with the starbursts being embedded in it. The age of the model agrees well with the star formation event in the window 400-500 Myr ago reported by McQuinn et al. (2010).

\subsubsection{Southern region (II)}

The best-fitting model for the southern region is characterized by a burst age of $3.9 \mathrm{Myr}$ with luminosity $5.3 \times 10^{41} \mathrm{erg} \mathrm{s}^{-1}$, density of $440 \mathrm{~cm}^{-3}$, and $r_{\text {in }} \simeq 22 \mathrm{pc}$. The ionization parameter is $\log (U)=-2.3$. Line predictions for the HII region and the PDR are shown in the bottom panel of Fig. 3. This burst found for the southern region is slightly younger than the burst in the central region, in agreement with Úbeda et al. (2007a). In the HII region, the [O III] $88 \mu \mathrm{m}$, [S III] 18.7 and $33.5 \mu \mathrm{m}$, and [S IV] $10.5 \mu \mathrm{m}$ lines are reproduced within $30 \%$, while [Ne III] $15.6 \mu \mathrm{m}$, [Ne II] $12.8 \mu \mathrm{m}$, and [N II] $122 \mu \mathrm{m}$ are underpredicted by a factor of $1.7,10$, and 6 , respectively. Feeding this model to the PDR, the [C II] $157 \mu \mathrm{m}$ line is underpredicted by a factor of 3.4, while the [O I] lines are both overpredicted by a factor of $\sim 2$. The contribution of the $\mathrm{H}$ II region to the PDR line 
Table 6. Observed and predicted MIR line ratios for the two star-forming regions.

\begin{tabular}{lccccc}
\hline \hline \multirow{2}{*}{ Ratio } & \multicolumn{3}{c}{ Central region } & \multicolumn{2}{c}{ Southern region } \\
& observed & burst & continuous & observed & burst \\
\hline$[\mathrm{Ne}$ III]/[Ne II] & 2.082 & 13.72 & 12.34 & 3.448 & 28.955 \\
{$[\mathrm{~S} \mathrm{IV}] /[\mathrm{S} \mathrm{III}] 18.7$} & 0.481 & 0.479 & 0.477 & 1.227 & 1.044 \\
{$[\mathrm{~S} \mathrm{III}] 18.7 /[\mathrm{S}$ III] $] 33$} & 0.630 & 0.593 & 0.601 & 0.835 & 0.846 \\
\hline
\end{tabular}

Notes. The $[\mathrm{Ne}$ III $] /[\mathrm{Ne}$ II $]$ and $[\mathrm{S} \mathrm{IV}] /[\mathrm{S} \mathrm{III}]$ line ratios are indicative of the radiation field strength, and the ratio of the two $[\mathrm{S}$ III] lines is a density diagnostic.

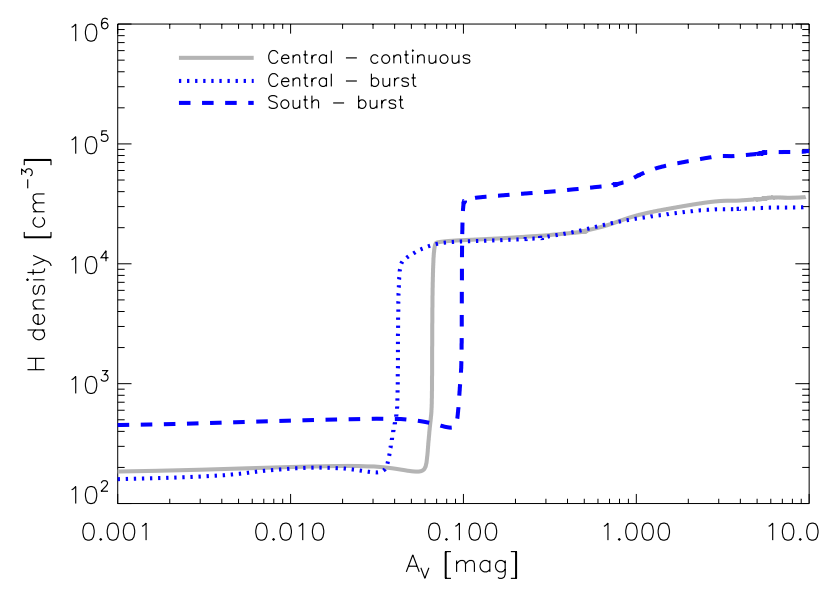

Fig. 4. Density profiles in the modeled clouds for the central and southern region, which include a turbulence pressure term $\left(v_{\text {turb }}=\right.$ $1.5 \mathrm{~km} \mathrm{~s}^{-1}$ ). Note that the $x$-axis is logarithmic, so the H II region (with a constant low density) occupies a thin layer of the cloud, stopping at low visual extinction $(\sim 0.1 \mathrm{mag})$.

emission is only $1 \%$. $G_{0}$ is found to be about $3.2 \times 10^{3}$, which is higher than in the central region. The luminosity of the model is 1.7 times higher than the observed $L_{\mathrm{TIR}}$ in this region.

\subsubsection{Comparison to empirical line ratios}

Physical conditions in the $\mathrm{HII}$ region are mainly determined by tracers of the radiation field strength ([Ne III]/ $[\mathrm{Ne}$ II $],[\mathrm{S} \mathrm{IV}] /[\mathrm{S} \mathrm{III}] 18.7 \mu \mathrm{m})$ and of density ([S III] $18.7 \mu \mathrm{m} /$ [S III] $33.5 \mu \mathrm{m})$. We compare these well-known diagnostic ratios in the two star-forming regions in Table 6 . In the southern region, ratios of $[\mathrm{Ne}$ III $] /[\mathrm{Ne}$ II $]$ and $[\mathrm{S} \mathrm{IV}] /[\mathrm{S} \mathrm{III}] 18.7 \mu \mathrm{m}$ are observed to be about twice as high and [S III] $18.7 \mu \mathrm{m} /[\mathrm{S}$ III] $33.5 \mu \mathrm{m}$ marginally higher than in the central region, indicating that the radiation field is harder and the medium denser. This is indeed what we recover with our best-fitting models (Table 4), as they match the sulfur ratios well.

\subsection{Effects of magnetic fields and turbulence on the PDR temperature and density}

After determining the best parameters for the $\mathrm{H}$ II regions, we explored the effect of cloud density on the PDR emission in more detail. Density is of critical importance in the emission output of the simulation because of the different critical densities of the observed lines. Density values quoted so far are representative of the $\mathrm{HII}$ region and evolve inside the modeled cloud. Figure 4 shows hydrogen density profiles in the clouds for each case presented in Sect. 4.1. The density starts at the initial value we set for each model in the $\mathrm{H}$ II region, remaining practically at the same level throughout the H II region. At the interface between the H II region and the PDR, there is a jump in density required to keep the model in pressure equilibrium. When pressure is only determined by the gas pressure $\left(P_{\text {gas }}=n_{\mathrm{H}} k T\right.$; i.e., magnetic fields and turbulence are omitted), the temperature difference at the phase transition is balanced by a rise in density of 2-3 orders of magnitude. Within this frame, the effects of magnetic fields or turbulence, implemented as pressure terms in CLOUDY, can be understood as follows: when total pressure equilibrium is assumed, they give more support at the phase transition, thus preventing a large difference in density between the $\mathrm{H}$ II region and the PDR and moderating the density increase at large optical depths. For the model presented in Sect. 4.1, where a low turbulence value is included but no magnetic fields, representative PDR densities are $2 \times 10^{4} \mathrm{~cm}^{-3}$ in the central region for both the single-burst and continuous star formation models, and $7 \times 10^{4} \mathrm{~cm}^{-3}$ in the southern region (see Fig. 4).

The effects of magnetic field and turbulence on the cloud density, temperature, and line emission are shown in Fig. 5. We present a set of runs for our best-fitting model in the central region single-burst case (note that we recover similar behaviors for the central continuous and southern single-burst cases, as shown in Appendix A) with the magnetic field $(B=30 \mu \mathrm{G})$ and/or the turbulence pressure $\left(v_{\text {turb }}=1.5,3,50 \mathrm{~km} \mathrm{~s}^{-1}\right)$ terms switched on. These terms have no impact on the ionic line emission because thermal pressure dominates the pressure balance in the HII region, but they noticeably change the emission of the PDR lines. When only thermal pressure is considered, the gas density jumps to values $>3 \times 10^{4} \mathrm{~cm}^{-3}$ in the PDR and the [OI] lines are overpredicted by an order of magnitude (black bars and dotted line). With only the magnetic field on, all three PDR lines are underpredicted by a factor of $\sim 3$ (orange bars), but their ratios are kept in the range observed thanks to the lower densities achieved $\left(2 \times 10^{3} \mathrm{~cm}^{-3}\right)$. Comparing models with different turbulent velocities, we see that the [C II] line is best matched for low or intermediate velocities because of their moderate densities $\left(\sim 10^{4} \mathrm{~cm}^{-3}\right)$ and slightly lower PDR temperatures (at $A_{\mathrm{V}} \simeq 1-3 \mathrm{mag}$ ). Increasing the turbulent velocity reduces the predicted [O I] emission and PDR density. The high-turbulence model performs poorly because it has the most dramatic effect on the density and line emission.

To summarize, we find that the best case that simultaneously matches all three PDR lines in the central region is the model with intermediate turbulent velocity $\left(v_{\text {turb }}=3 \mathrm{~km} \mathrm{~s}^{-1}\right)$, which has a density of $8 \times 10^{3} \mathrm{~cm}^{-3}$, but we stress that the main effect of turbulence is to reduce the PDR density.

\subsection{Input spectra and SED}

Figure 6 shows the input and output SEDs of the models for the two regions. The input SED is the stellar spectrum of the illuminating source modeled with STARBURST99 and also includes the $\mathrm{CMB}$ at millimeter wavelengths. In the central region, the stellar spectrum has a wider distribution for the continuous 

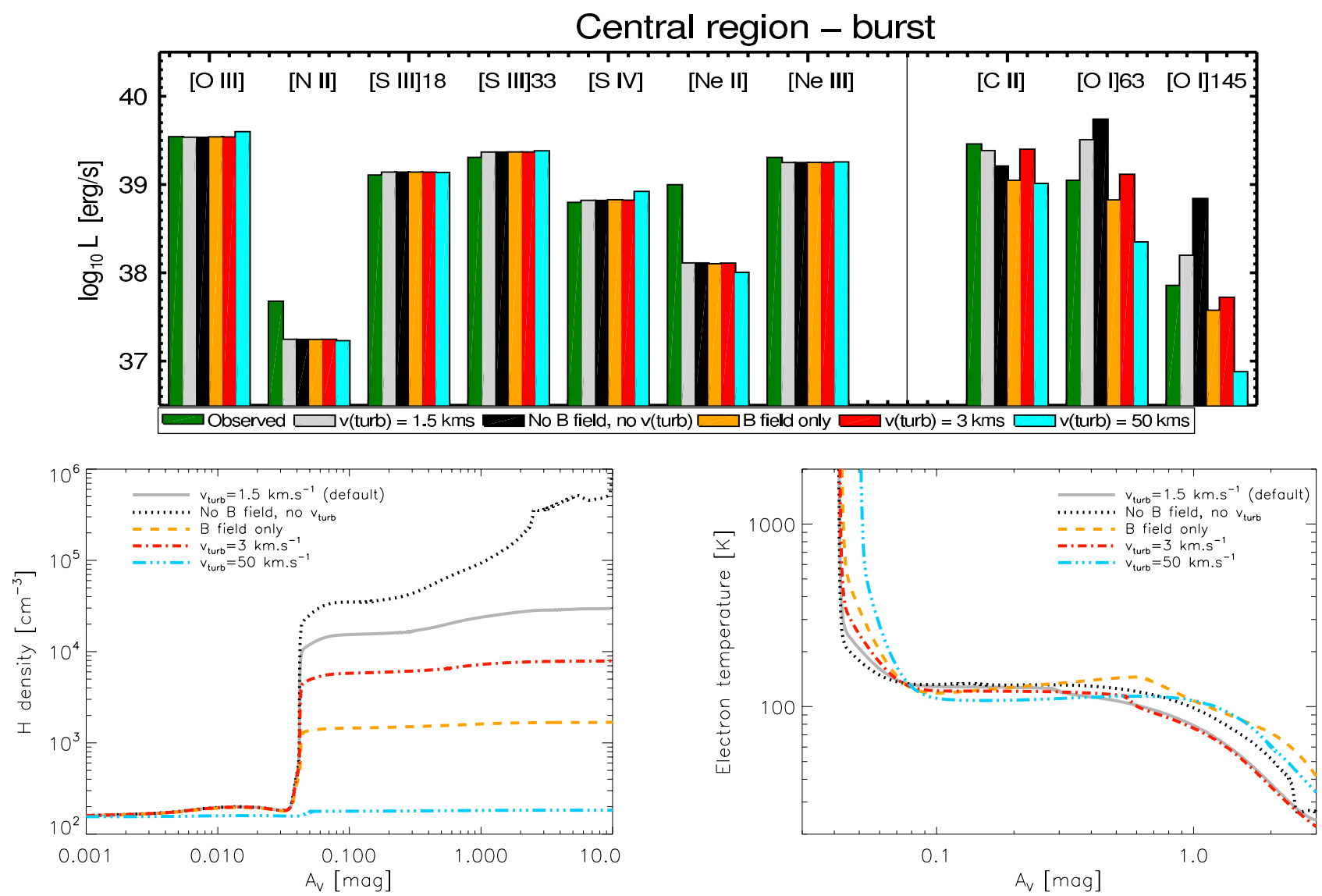

Fig. 5. Effect of turbulence and magnetic fields on the predicted line intensities (top panel), density, and temperature (bottom panels) in the modeled cloud for the central region single-burst case. Green bars: observations. Gray bars and solid lines: only low turbulence switched on $\left(v_{\text {turb }}=1.5 \mathrm{~km} \mathrm{~s}^{-1}\right.$, default model). Black bars and dotted lines: no magnetic fields and no turbulence. Orange bars and dashed lines: only magnetic field switched on $(B=30 \mu \mathrm{G})$. Red bars and dash-dotted line: only moderate turbulence switched on $\left(v_{\text {turb }}=3 \mathrm{~km} \mathrm{~s}^{-1}\right)$. Cyan bars and tripledotted-dashed line: only high turbulence switched on $\left(v_{\text {turb }}=50 \mathrm{~km} \mathrm{~s}^{-1}\right)$.

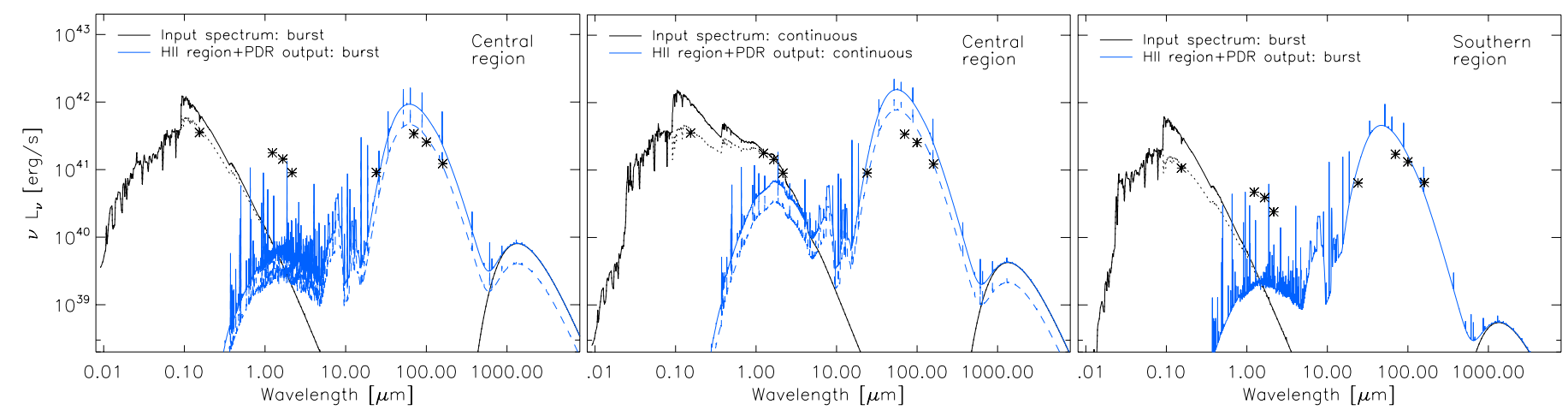

Fig. 6. Spectral energy distributions of the two star-forming regions: central region single-burst case (left panel), central region continuous case (middle panel), and southern region single-burst model (right panel). The black and blue curves correspond to the input and output SEDs, respectively. The dotted lines are the attenuated input SEDs. The data points are the photometry measurements from GALEX FUV, 2 MASS $J, H$, $K$ bands, MIPS $24 \mu \mathrm{m}$, and PACS at $70 \mu \mathrm{m}, 100 \mu \mathrm{m}$, and $160 \mu \mathrm{m}$. In panels for the central region, the dashed curves are scaled versions of the output SEDs, considering a covering factor of 0.5 for the PDR.

star formation model than the single-burst star formation model (black curves), and it is more luminous in the near-IR regime due to the presence of old stars. Compared to observations, all input SEDs fall above the GALEX FUV data because they are unattenuated, and the single-burst input SEDs fall below the 2MASS data because they lack old stars. In the central region, the input SED of the continuous model, on the other hand, agrees well with the 2MASS data. For better agreement with the FUV data, we estimated the level of extinction required to attenuate the input SEDs. We considered average extinction values $E(B-V)$ of $0.1,0.05$, and $0.1 \mathrm{mag}$ for the central continuous, central single-burst, and southern single-burst models, respectively (dotted lines in Fig. 6), which are in the range of values found by Úbeda et al. (2007b).

Focusing on the output SEDs of the models for the central region, it is anticipated that the level of the FIR continuum is 
different. The higher the input luminosity of the source, the higher the peak of the output SED. Moreover, as the dust temperature rises, the peak of the output SED is expected to shift to shorter wavelengths. For the continuous model, the higher FUV luminosity therefore provides more dust heating, explaining the slight shift of its peak to shorter wavelengths. We can compare the output SEDs to the observed PACS $70 \mu \mathrm{m}$, $100 \mu \mathrm{m}$, and $160 \mu \mathrm{m}$ fluxes. The models agree relatively well with observations for the southern region, but overpredict the FIR continuum emission in the central region. This is not surprising because we used as input luminosity a higher value than the observed TIR flux and the modeled PDR has a high $A_{\mathrm{V}}$. Better agreement with continuum observations requires a model that predicts a TIR flux lower by a factor of 2-3, for example, by reducing the covering factor of the PDR. We return to this in Sect. 5.1.3.

\section{Discussion}

We have presented models for the two star-forming regions of NGC 4214 that work for most of the observed MIR and FIR lines. Some discrepancies remain between our models and observations ([Ne II], [N II], [C II ]/[OI], and $L_{\mathrm{TIR}}$ ); these are not due to the choice of parameter space but rather to missing physics or components in our models. In this section, we discuss various aspects of our analysis: 1) the discrepancies with observations, and we give clues to improve our models; 2) which star formation scenario describes the data better; and 3) how our ISM results relate to the known properties and evolution of the two regions.

\subsection{Discrepancies between models and observations}

\subsubsection{Ionic lines}

In our best-fitting models of the H II region, the [Ne II] $12.8 \mu \mathrm{m}$ and [N II] $122 \mu \mathrm{m}$ lines are systematically underpredicted. These lines have lower excitation potentials $(21.56$ and $14.53 \mathrm{eV}$, respectively) than the other, better matched ionic lines, and can therefore be partially excited outside of the main $\mathrm{H}$ II region. This effect can be significant in NGC 4214 because of the poor spatial resolution.

Discrepancies between the observed and modeled $[\mathrm{Ne} \mathrm{III}] /[\mathrm{Ne} \mathrm{II}]$ ratio, with the same amplitude as we found for NGC 4214, were reported by Martín-Hernández et al. (2002) for $\mathrm{H}$ II regions observed by ISO/SWS (see their Fig. 2). These could again be related to a mixture of physical conditions within the ISO beam, which is also relatively large. For the starburst galaxy Haro 11, we have examined the effect of an additional low-ionization component (star of effective temperature $35000 \mathrm{~K}$ and $n_{\mathrm{H}} \simeq 10^{1-3} \mathrm{~cm}^{-3}$ ). This reproduced the observed [Ne II] $12.8 \mu \mathrm{m}$ and [N II] $122 \mu \mathrm{m}$ emission without significantly affecting the other ionic lines (Cormier et al. 2012). Alternatively, given that the neon lines have the largest energy difference, they are more sensitive to the underlying stellar atmosphere models than the sulfur lines. Constraining those models is beyond the scope of this paper, and we relied on the sulfur lines as being more robust diagnostics of the $\mathrm{H}$ II region conditions in NGC 4214 (i.e., models presented in Sect. 4.1).

We also assessed the effect of including the [Ne II] $12.8 \mu \mathrm{m}$ line in the best-fitting solution-tracking procedure for the southern region. This resulted in a solution where the [Ne II] $12.8 \mu \mathrm{m}$ absolute flux was better reproduced (within a factor of 3). This model uses an older burst (5 Myr), higher density $\left(500 \mathrm{~cm}^{-3}\right)$, and the same inner radius $(20 \mathrm{pc})$. The corresponding ionization parameter is $\log (U)=-2.4$, which is 0.1 dex lower than previously found. However, the model underpredicts the neon intensities by a factor of 2 and the [S IV]/[S III] $18.7 \mu \mathrm{m}$ ratio by a factor of $\sim 4$, while that ratio was matched within $20 \%$ without the $[\mathrm{Ne} \mathrm{II}]$ constraint.

\subsubsection{PDR lines}

In our default PDR solutions for both regions, [C II] is systematically underpredicted compared to [O I]. The best PDR model, found in Sect. 4.2 for the central region single-burst case, includes a turbulent velocity of $3 \mathrm{~km} \mathrm{~s}^{-1}$. In the southern and central region continuous case, similar turbulent velocities also lead to better agreement with the $[\mathrm{CII}] /[\mathrm{OI}]$ line ratios, but still underpredict the observed emission in absolute values (Appendix A).

In addition to the stars, X-rays can be a source of heating in the PDR and affect the FIR line emission. Point sources and diffuse X-ray emission have been reported in Hartwell et al. (2004) and Ghosh et al. (2006). The identified point sources are not coincident with the peak of the FIR emission, and we therefore ignored them. The diffuse emission is mostly detected in the central region, with a luminosity of $3 \times 10^{38} \mathrm{erg} \mathrm{s}^{-1}$ (Hartwell et al. 2004), which is lower than that of the starburst. We have tested the effect of this diffuse X-ray component on the PDR lines in the central region and found that it increases the predicted intensity of the [O I] lines by $\sim 30 \%$ and the [C II] intensity by less than $10 \%$. As X-rays are not the main source of heating in the PDR, they do not help to produce significantly more [C II] emission.

We further explored the possibility of [C II] originating from a diffuse ionized component. We compared the [C II] and [N II] $122 \mu \mathrm{m}$ intensities and the PACS upper limit on the [N II] $205 \mu \mathrm{m}$ line, which gives [N II] $122 \mu \mathrm{m} /[\mathrm{N} \mathrm{II}] 205 \mu \mathrm{m}>1$, to theoretical predictions assuming pure collisional regime. Following Bernard-Salas et al. (2012) and applying $C$ and $N$ elemental abundances observed in NGC 4214, we found that less than $16 \%$ of the total observed [C II] emission arises in diffuse ionized gas. Herschel SPIRE FTS observations of [N II] $205 \mu \mathrm{m}$ toward the central region also indicate that [N II] $122 \mu \mathrm{m} /[\mathrm{N} \mathrm{II}]$ $205 \mu \mathrm{m}=2.5$ (priv. comm. R. Wu), which gives an ionized gas density of $\sim 60 \mathrm{~cm}^{-3}$ and a contribution of only $8 \%$ to the total [C II] emission. Therefore, if a low-density ionized component is added to our current models to account for the missing [N II] and [Ne II] emission, this component will not contribute significantly to the [C II] emission. Our best-fitting models for the central region also predict that the $\mathrm{H}$ II region contributes less than a few percent to the $[\mathrm{C}$ II] emission.

The $[\mathrm{CII}]$ emission most likely arises from a neutral phase, but its conditions are not well described by our default PDR models. With the PDR tests performed in Sect. 4.2, we have explored the effect of density on the PDR emission lines, but these lines are also sensitive to the radiation field strength. To reduce the radiation field intensity $G_{0}$ (not the hardness), we placed the PDR farther away by stopping the model at the $\mathrm{H}^{+}$/ $\mathrm{H}$ I phase transition and resuming the calculation at a larger distance in the H I phase (note that this breaks the pressure equilibrium). The direct effect is to dilute the UV field before it reaches the PDR, as proposed by Israel \& Maloney (2011) and Cormier et al. (2015), which is equivalent to increasing the porosity of the 


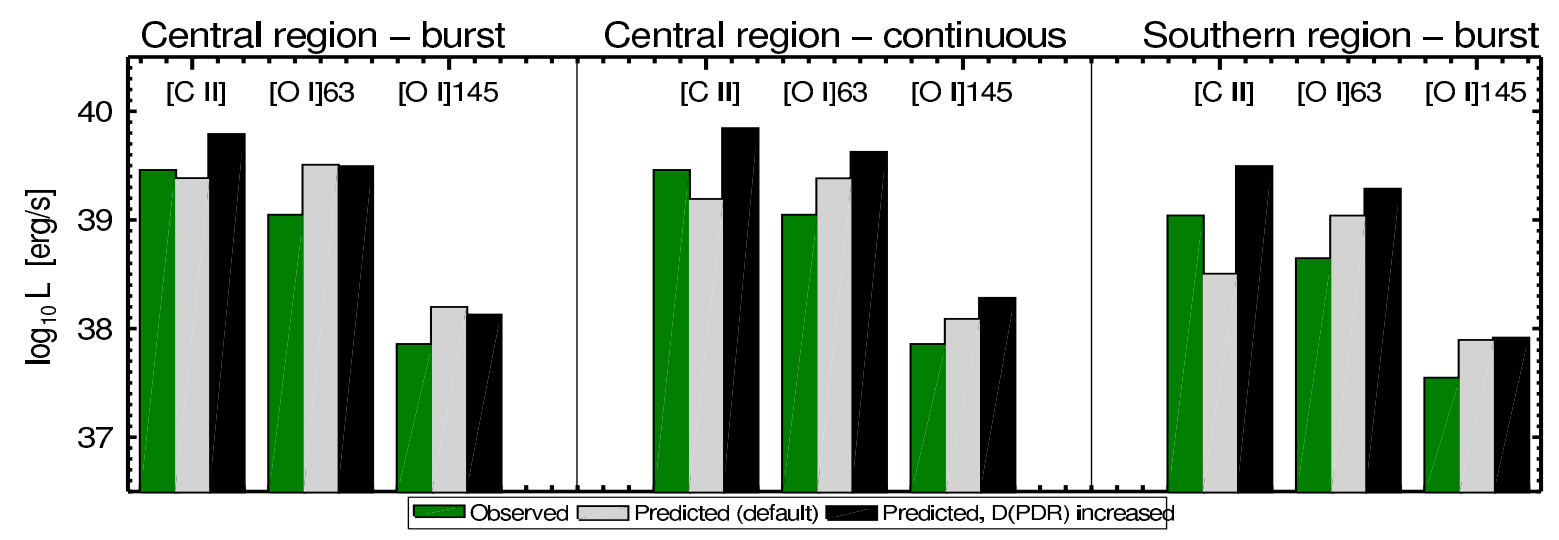

Fig. 7. Effect of changing the distance to the modeled cloud for the PDR calculation. The PDR distance is increased by a factor of 2, 3, and 5 for the central single-burst (left panel), central continuous (middle panel), and southern single-burst cases (right panel), respectively.

medium. For the central single-burst, central continuous, and the southern single-burst cases, we increased the PDR distance by a factor of 2,3 , and 5 ( $r_{\text {in }} \simeq 170,186$, and $\left.115 \mathrm{pc}\right)$, respectively. This way, $G_{0}$ decreases to $\sim 120$ in all three cases, boosting the [C II] emission, and the predicted [C II]/[O I] ratios match the observed ratios within $40 \%$ (Fig. 7). In absolute values, the [C II] and $[\mathrm{OI}$ ] intensities are 2 to 3 times too high, however. This can be compensated by a PDR covering factor lower than unity (see Sect. 5.1.3 below).

We conclude that a moderate density, moderate $G_{0}$ neutral medium (compared to our dense, high $G_{0}$ default PDR model) with a low turbulent velocity and a covering factor lower than unity is the most plausible origin for the observed [C II] emission.

\subsubsection{Model luminosity}

Hermelo et al. (2013) have fit the dust SED of the whole galaxy. In particular, their models require less UV luminosity than that observed to match the IR emission. They discussed possible explanations for this disagreement, proposing an escape of unattenuated UV radiation along with a particular geometry and dust properties in the galaxy. Such an argument of a UV escape fraction could also apply in our case. As seen in Fig. 6, the observed photometry data points in the FIR, which correspond to emission originating from the PDR, are lower than the modeled SED for the central region. This disagreement can indicate a different covering factor for the PDR. In our model, the PDR fully covers the sphere around the source (i.e., covering factor of unity). For the best-fitting models to better match the photometry, it could be that the PDR component is more porous, allowing radiation to escape the cloud. To illustrate this, we plot the resulting SEDs for the two models of the central region considering a PDR covering factor of 0.5 (dashed curves in Fig. 6).

Part of the discrepancies in our results that we have discussed in this Sect. 5.1 originate in modeling each complex as a single cloud, which is imposed by the lack of spatial resolution in the observations. Clearly, future improvements are expected from observations with better spatial resolution.

\subsection{Central region: bursty or continuous star formation?}

The star formation history of NGC 4214 over the last Gyr is complex. It shows bursts lasting for shorter or longer periods and a continuous "background", which takes place throughout this whole time window (McQuinn et al. 2010; Williams et al. 2011). In that sense, we could say that it is a rather hybrid star formation pattern. In the central region, we have investigated cases of both a single-burst and a continuous star formation mode. However, when we use a single model to reproduce the observed line emission, we simplify the problem, since we do not take into account both modes. Hence arises the question of which of the two approaches is the most adequate to model the MIR-FIR line emission. We have shown that both modes can satisfyingly reproduce the observed mid- and far-infrared line emission. By comparing the $\bar{\chi}^{2}$ values of the best-fitting models (Table 5), we found that the single-burst case seems to globally perform better when considering the lines used in the optimization method. When including [N II] and [Ne II], the two modes perform similarly, although the high $\bar{\chi}^{2}$ values are driven by the poor fit to the [Ne II] $12.8 \mu \mathrm{m}$ line. For the PDR, the continuous scenario gives a lower $\bar{\chi}^{2}$, but the default PDR solutions are not optimum and can be fine-tuned for both modes (by lowering the density and $G_{0}$, see Sects. 4.2 and 5.1). We conclude that both are limiting, simplifying cases of modeling the ISM in NGC 4214, with the continuous star formation model being marginally more accurate inside the PDR and the single-burst in the $\mathrm{HII}$ region (without further refinement).

\subsection{Comparison between the two star-forming regions}

How do the ISM conditions that we have characterized in the two star-forming regions relate to their star formation properties? We have found that the modeled cluster (radiation source) in the southern region contains younger stellar populations with a harder radiation field than that in the central region, in agreement with the results of Úbeda et al. (2007a), for instance. The hydrogen density is also higher in the southern star-forming region, but the metallicities of the regions are very similar. The southern region is observed to be at a younger, more compact stage than the central region. The central region is more evolved and had time to expand, as observed by the presence of shells that may have swept away the dense material (Walter et al. 2001), and is thus consistent with a more diffuse ISM.

We calculated the star formation rate surface densities for the two regions combining the GALEX FUV map and the Spitzer $24 \mu \mathrm{m}$ map, as done in Leroy et al. (2008), with

$$
\begin{aligned}
\Sigma_{\mathrm{SFR}}\left[M_{\odot} \mathrm{yr}^{-1} \mathrm{kpc}^{-2}\right]= & 3.2 \times 10^{-3} \cdot I_{24}\left[\mathrm{MJy} \mathrm{sr}^{-1}\right] \\
& +8.1 \times 10^{-2} \cdot I_{\mathrm{FUV}}\left[\mathrm{MJy} \mathrm{sr}^{-1}\right],
\end{aligned}
$$


Table 7. Comparison of star formation properties.

\begin{tabular}{lcc}
\hline \hline Quantity & Region I & Region II \\
\hline$L_{\mathrm{CII}} / L_{\mathrm{TIR}}$ & $5.4 \times 10^{-3}$ & $3.6 \times 10^{-3}$ \\
$L_{\mathrm{CII}} / L_{\mathrm{CO}(1-0)}$ & $6.7 \times 10^{4}$ & $2.5 \times 10^{4}$ \\
$M_{\mathrm{gas}=\mathrm{H}_{2}+\mathrm{HI}}\left[M_{\odot}\right]$ & $2.05 \times 10^{6}$ & $2.68 \times 10^{6}$ \\
$M_{\mathrm{H}_{2}} / M_{\mathrm{HI}}$ & 0.35 & 0.32 \\
$S F R\left[M_{\odot} \mathrm{yr}^{-1}\right]$ & $2.2 \times 10^{-2}$ & $1.9 \times 10^{-2}$ \\
$S F E\left[\mathrm{Gyr}^{-1}\right]$ & 10.7 & 7.1 \\
\hline
\end{tabular}

where $\Sigma_{\text {SFR }}$ is the SFR surface density and $I_{24}$ and $I_{\mathrm{FUV}}$ the $24 \mu \mathrm{m}$ and FUV intensities. We also measured the atomic and molecular hydrogen content of the two regions using the $21 \mathrm{~cm}$ map from THINGS ${ }^{2}$ (Walter et al. 2008) and the CO(1-0), CO(21) transition maps from Walter et al. (2001) and HERACLES 3 (Leroy et al. 2009), respectively. We used a conversion factor of $\alpha_{\mathrm{CO}}=4.38\left[M_{\odot} \mathrm{pc}^{-1}\left(\mathrm{~K} \mathrm{~km} \mathrm{~s}^{-1}\right)^{-1}\right]$ from $\mathrm{CO}(1-0)$ luminosity to $\mathrm{H}_{2}$ mass. If we were to use a different conversion factor due to the low metallicity of these regions (e.g., Schruba et al. 2012), this would not affect the relative comparison of the regions (see Table 7). The central region (I) has a total hydrogen content of $M_{\text {gas, } \mathrm{I}}=M_{\mathrm{HI}}+M_{\mathrm{H}_{2}}=2.05 \times 10^{6} M_{\odot}$ and a molecular (mass) fraction of $f_{\mathrm{mol}, \mathrm{I}}=M_{\mathrm{H}_{2}} / M_{\mathrm{HI}}=0.35$. Integrating Eq. (3) in the region, we find $S F R_{\mathrm{I}}=2.2 \times 10^{-2} M_{\odot} \mathrm{yr}^{-1}$. The southern region (II) has a higher total hydrogen content of $M_{\text {gas,II }}=2.68 \times 10^{6} M_{\odot}$, an $\mathrm{H}_{2}$ fraction $f_{\text {mol,II }}=0.32$, and $S F R_{\mathrm{II}}=1.9 \times 10^{-2} M_{\odot} \mathrm{yr}^{-1}$. Therefore the southern region has relatively more gas compared to its SFR than the central region. In terms of efficiency, SFR $/ M_{\text {gas }}$, it is about $50 \%$ lower in the southern region. This could reflect a slightly more efficient, cluster-like star formation episode in the central region or simply encode a different evolutionary state as the SFR and gas masses are a strong function of time on scales of individual star-forming regions (e.g., Schruba et al. 2010). The southern region being younger, it may still be in the process of forming stars.

The main differences in ISM conditions that we extracted from our modeling relate to the $\mathrm{H}$ II region properties. The emission lines are a factor of two lower in luminosity in the southern region, except [Ne III] and [S IV], which are proportionally higher in the southern region. By contrast, the PDR conditions in the two regions are similar $\left(n_{\mathrm{H}} \simeq 10^{4} \mathrm{~cm}^{-3}\right.$ and $\left.G_{0} \simeq 150\right)$. Our modeling reflects conditions resulting from the recent starforming event and has little predictive power regarding a different, future star-forming event. In particular, at the linear scale that we probe $(\sim 175 \mathrm{pc})$, the PDR conditions are averaged over multiple star-forming clouds and not representative of the underlying, possibly different, substructure in individual molecular clouds. However, there is more PDR emission relative to $\mathrm{CO}$ in the central region, that is, high $[\mathrm{C} \mathrm{II}] / \mathrm{CO}$ and $[\mathrm{O} \mathrm{I}] / \mathrm{CO}$ ratios (see also Cormier et al. 2010). As found by Walter et al. (2001), $\mathrm{CO}$ emission is centrally concentrated in the south and more diffuse in the center. The concentration of molecular gas may be nourishing the current star formation episode in the south or is being observed at a pre-disruption stage with the same fate as the central region. The increased porosity, which evidently is an intrinsic property of the low-metallicity ISM, is seen in both the central and southern star-forming regions. The main evolution within the dense medium is seen in the covering factor of the PDR, which is found to be lower in the central, more evolved

\footnotetext{
2 http://www.mpia-hd.mpg.de/THINGS/Data.html

3 http://www.cv.nrao.edu/ aleroy/heracles_data/
}

region than in the southern region, and in $\mathrm{CO}$, which probably suffers more from photodissociation with time and its emission is seen farther away from the cluster center, but this cannot be modeled with our static approach. Observing an intermediate PDR tracer at the $\mathrm{C}^{+} / \mathrm{CO}$ transition, such as $\mathrm{C}$, would help to test this evolution scenario.

\section{Conclusion}

We have investigated the physical conditions characterizing the ISM of the dwarf irregular galaxy NGC 4214 by modeling Spitzer and Herschel observations of MIR and FIR fine-structure cooling lines. We used the spectral synthesis code CLOUDY to self-consistently model the HII region and PDR properties of the two main star-forming regions in NGC 4214. We summarize our results as follows:

- The ionized gas in the southern region is found to be 2.5 times denser than in the central region $\left(440 \mathrm{~cm}^{-3}\right.$ versus $170 \mathrm{~cm}^{-3}$ ) and typified by a harder radiation field. Our best-fitting models of the $\mathrm{H}$ II region+PDR reproduce most ionic and neutral atomic lines, namely the [O III] $88 \mu \mathrm{m}$, [S III] 18.7 and $33.5 \mu \mathrm{m}$, [S IV] $10.5 \mu \mathrm{m}$, [Ne III] $15.6 \mu \mathrm{m}$, and $[\mathrm{OI}] 63 \mu \mathrm{m}$ lines, within a factor of $\sim 2$.

- The observed [N II] $122 \mu \mathrm{m}$ and [Ne II] $12.8 \mu \mathrm{m}$ lines are the most discrepant with our model solutions for the H II region. A single model component seems too simplistic to account for all observed lines simultaneously. Given the complexity of these star-forming regions, a multi-component modeling would be more appropriate. In particular, a lower excitation ionized gas component may be required to match the [N II] and $[\mathrm{Ne}$ II] emission in both regions.

- Our HII region models and the established observational $[\mathrm{C} \mathrm{II}] /[\mathrm{N} \mathrm{II}]$ line ratio used as a proxy for the fraction of [C II] arising in the ionized gas both indicate that the [C II] emission is mostly associated with the PDR gas, with only a $\sim 10 \%$ contribution from the ionized gas.

- Constant pressure models where thermal pressure dominates the pressure equilibrium perform rather poorly for the PDR lines mostly because of the high densities and high $G_{0}$ values reached in the PDR. Including additional pressure terms, such as weak turbulent or magnetic pressure, or placing the PDR cloud farther away and reducing its covering factor, leads to a much improved reproduction of the observed line intensities.

- Star formation histories have an effect on the predicted MIR-FIR line emission. We have explored the two simplifying cases of a bursty and a continuous star formation scenario. In the central region, we found that the bursty scenario works marginally better for the H II region and the continuous scenario for the PDR, although both modes can reproduce the observations after refining the PDR conditions.

- The H II region modeling from IR emission is consistent with the evolutionary stages of the regions found in previous studies: the southern region is younger and more compact, while the central region is more evolved and diffuse. On the linear scale of our study $(\sim 175 \mathrm{pc})$, the PDR conditions of individual star-forming clouds are averaged out and do not echo the observed differences between the two regions (stellar ages, H II conditions, etc.). The increased porosity of the star-forming regions appears as an intrinsic characteristic of the low-metallicity ISM, while the covering factor of the PDR, which is reduced in the central region, stands out as the main evolution tracer. 

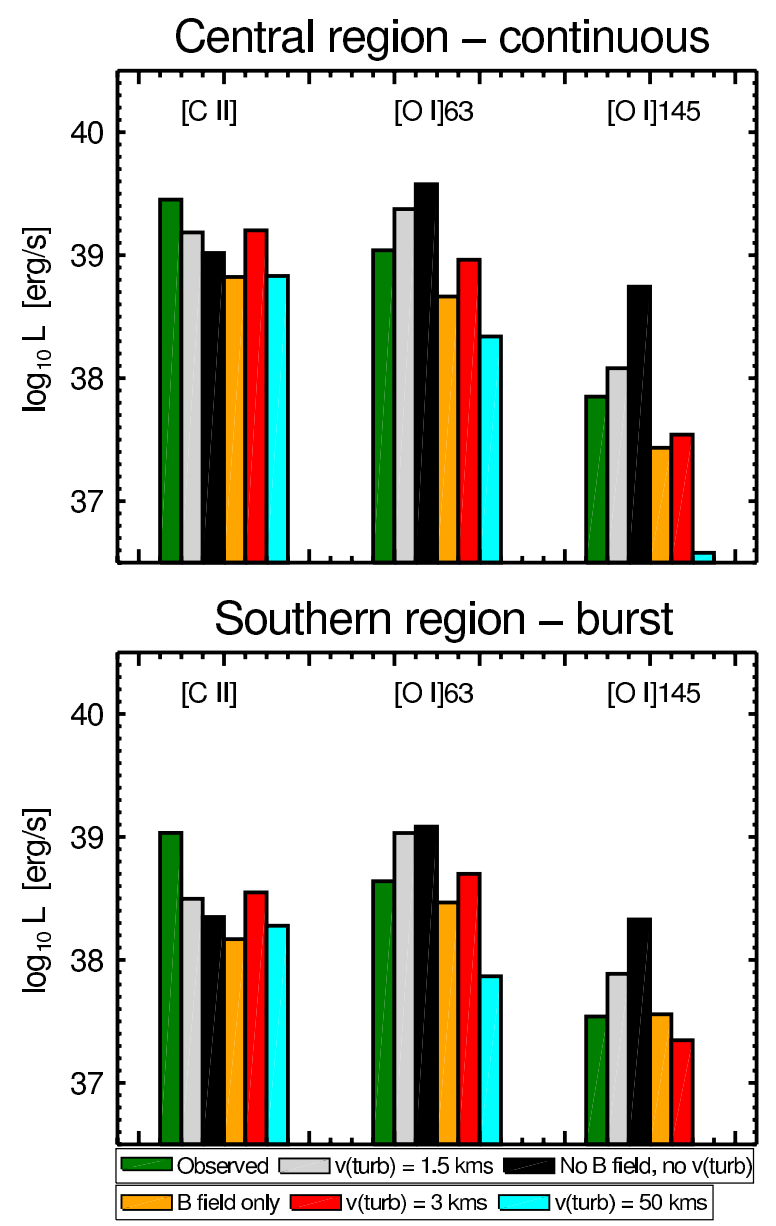

Fig. A.1. Same as Fig. 5 for the central region continuous case (top panel) and for the southern region single-burst (bottom panel). See Sect. 4.2 for details.

Acknowledgements. We would like to thank S. Hony for his help with the IRS data and for fruitful discussion, F. Walter for providing us the $\mathrm{CO}(1-0)$ data, and S. Glover for his advice on turbulence issues. We thank the referee for his or her comments on the manuscript. D.C. and F.B. acknowledge support from DFG grant BI 1546/1-1. This work is based in part on archival data obtained with the Spitzer Space Telescope, which is operated by the Jet Propulsion Laboratory, California Institute of Technology under a contract with NASA. Herschel is an ESA space observatory with science instruments provided by European-led Principal Investigator consortia and with important participation from NASA.

\section{Appendix A: Comparison of PDR model predictions and observations}

We show in this appendix the effect of turbulence and magnetic fields on the predicted PDR line intensities for the central region continuous case and for the southern region single-burst case. Tests with turbulence and magnetic fields are presented in Sect. 4.2 only for the central region single-burst case.

\section{References}

Abel, N. P., Ferland, G. J., Shaw, G., \& van Hoof, P. A. M. 2005, ApJS, 161, 65 Andrews, J. E., Calzetti, D., Chandar, R., et al. 2013, ApJ, 767, 51

Aniano, G., Draine, B. T., Gordon, K. D., \& Sandstrom, K. 2011, PASP, 123, 1218

Asplund, M., Grevesse, N., Sauval, A. J., \& Scott, P. 2009, ARA\&A, 47, 481 Bendo, G. J., Galliano, F., \& Madden, S. C. 2012, MNRAS, 423, 197
Bernard-Salas, J., Habart, E., Arab, H, et al. 2012, A\&A, 538, A37 Bernard-Salas, J., Habart, E., Köhler, M., et al. 2015, A\&A, 574, A97 Cormier, D., Madden, S. C., Hony, S., et al. 2010, A\&A, 518, L57

Cormier, D., Lebouteiller, V., Madden, S. C., et al. 2012, A\&A, 548, A20

Cormier, D., Madden, S. C., Lebouteiller, V., et al. 2015, A\&A, 578, A53

Cubick, M., Stutzki, J., Ossenkopf, V., Kramer, C., \& Röllig, M. 2008, A\&A, 488,623

Dalcanton, J. J., Williams, B. F., Seth, A. C., et al. 2009, ApJS, 183, 67

Dale, D. A., Smith, J. D. T., Schlawin, E. A., et al. 2009, ApJ, 693, 182

Ferland, G. J., Porter, R. L., van Hoof, P. A. M., et al. 2013, Rev. Mex. Astron. Astrofis., 49, 137

Galametz, M., Kennicutt, R. C., Calzetti, D., et al. 2013, MNRAS, 431, 1956

Galliano, F., Madden, S. C., Tielens, A. G. G. M., Peeters, E., \& Jones, A. P. 2008, ApJ, 679, 310

Ghosh, K. K., Rappaport, S., Tennant, A. F., et al. 2006, ApJ, 650, 872

Graciá-Carpio, J., Sturm, E., Hailey-Dunsheath, S., et al. 2011, ApJ, 728, L7

Habing, H. J. 1968, Bull. Astron. Inst. Netherlands, 19, 421

Hartwell, J. M., Stevens, I. R., Strickland, D. K., Heckman, T. M., \& Summers, L. K. 2004, MNRAS, 348, 406

Heiles, C. 1994, ApJ, 436, 720

Hermelo, I., Lisenfeld, U., Relaño, M., et al. 2013, A\&A, 549, A70

Houck, J. R., Roellig, T. L., Van Cleve, J., et al. 2004, in SPIE Conf. Ser. 5487, ed. J. C. Mather, 62

Hunt, L. K., Thuan, T. X., Izotov, Y. I., \& Sauvage, M. 2010, ApJ, 712, 164

Hunter, D. A., Kaufman, M., Hollenbach, D. J., et al. 2001, ApJ, 553, 121

Hunter, D. A., Ficut-Vicas, D., Ashley, T., et al. 2012, AJ, 144, 134

Israel, F. P., \& Maloney, P. R. 2011, A\&A, 531, A19

Izotov, Y. I., \& Thuan, T. X. 1999, ApJ, 511, 639

Kaufman, M. J., Wolfire, M. G., \& Hollenbach, D. J. 2006, ApJ, 644, 283

Kepley, A. A., Zweibel, E. G., Wilcots, E. M., Johnson, K. E., \& Robishaw, T. 2011, ApJ, 736, 139

Kobulnicky, H. A., \& Skillman, E. D. 1996, ApJ, 471, 211

Kroupa, P. 2001, MNRAS, 322, 231

Kunth, D., \& Östlin, G. 2000, A\&ARv, 10, 1

Lebouteiller, V., Cormier, D., Madden, S. C., et al. 2012, A\&A, 548, A91

Leitherer, C., Ortiz Otálvaro, P. A., Bresolin, F., et al. 2010, ApJS, 189, 309

Leroy, A. K., Walter, F., Brinks, E., et al. 2008, AJ, 136, 2782

Leroy, A. K., Walter, F., Bigiel, F., et al. 2009, AJ, 137, 4670

Madden, S. C., Galliano, F., Jones, A. P., \& Sauvage, M. 2006, A\&A, 446, 877

Madden, S. C., Rémy-Ruyer, A., Galametz, M., et al. 2013, PASP, 125, 600

Martín-Hernández, N. L., Vermeij, R., Tielens, A. G. G. M., van der Hulst, J. M., \& Peeters, E. 2002, A\&A, 389, 286

McIntyre, V. J. 1998, PASA, 15, 157

McKee, C. F., \& Ostriker, E. C. 2007, ARA\&A, 45, 565

McQuinn, K. B. W., Skillman, E. D., Cannon, J. M., et al. 2010, ApJ, 721, 297

Mookerjea, B., Kramer, C., Buchbender, C., et al. 2011, A\&A, 532, A152

Osterbrock, D. E., \& Ferland, G. J. 2006, Astrophysics of gaseous nebulae and active galactic nuclei (Sausalito, CA: University Science Books)

Ott, S. 2010, in Astronomical Data Analysis Software and Systems XIX, eds. Y. Mizumoto, K.-I. Morita, \& M. Ohishi, ASP Conf. Ser., 434, 139

Parkin, T. J., Wilson, C. D., Schirm, M. R. P., et al. 2013, ApJ, 776, 65

Pilbratt, G. L., Riedinger, J. R., Passvogel, T., et al. 2010, A\&A, 518, L1

Poglitsch, A., Waelkens, C., Geis, N., et al. 2010, A\&A, 518, L2

Rémy-Ruyer, A., Madden, S. C., Galliano, F., et al. 2013, A\&A, 557, A95

Röllig, M., Abel, N. P., Bell, T., et al. 2007, A\&A, 467, 187

Rubin, R. H., Simpson, J. P., Lord, S. D., et al. 1994, ApJ, 420, 772

Schruba, A., Leroy, A. K., Walter, F., Sandstrom, K., \& Rosolowsky, E. 2010, ApJ, 722, 1699

Schruba, A., Leroy, A. K., Walter, F., et al. 2012, AJ, 143, 138

Smith, J. D. T., Armus, L., Dale, D. A., et al. 2007, PASP, 119, 1133

Sollima, A., Gratton, R. G., Carretta, E., Bragaglia, A., \& Lucatello, S. 2013, MNRAS, 433, 1276

Sollima, A., Cignoni, M., Gratton, R. G., et al. 2014, MNRAS, 437, 1918

Tielens, A. G. G. M., \& Hollenbach, D. 1985, ApJ, 291, 722

Úbeda, L., Maíz-Apellániz, J., \& MacKenty, J. W. 2007a, AJ, 133, 917

Úbeda, L., Maíz-Apellániz, J., \& MacKenty, J. W. 2007b, AJ, 133, 932

Vasta, M., Barlow, M. J., Viti, S., Yates, J. A., \& Bell, T. A. 2010, MNRAS, 404, 1910

Verma, A., Lutz, D., Sturm, E., et al. 2003, A\&A, 403, 829

Walter, F., Taylor, C. L., Hüttemeister, S., Scoville, N., \& McIntyre, V. 2001, AJ, 121,727

Walter, F., Brinks, E., de Blok, W. J. G., et al. 2008, AJ, 136, 2563

Werner, M. W., Roellig, T. L., Low, F. J., et al. 2004, ApJS, 154, 1

Wilcots, E. M., \& Thurow, J. C. 2001, ApJ, 555, 758

Williams, B. F., Dalcanton, J. J., Gilbert, K. M., et al. 2011, ApJ, 735, 22

Wu, Y., Charmandaris, V., Houck, J. R., et al. 2008, ApJ, 676, 970 